\title{
Compacts connexes invariants par une application univalente
}

\author{
par
}

\author{
Emmanuel Risler (Nice)
}

\begin{abstract}
Let $K$ be a compact connected subset of $\mathbb{C}$, not reduced to a point, and $F$ a univalent map in a neighborhood of $K$ such that $F(K)=K$. This work presents a study and a classification of the dynamics of $F$ in a neighborhood of $K$. When $\mathbb{C} \backslash K$ has one or two connected components, it is proved that there is a natural rotation number associated with the dynamics. If this rotation number is irrational, the situation is close to that of "degenerate Siegel disks" or "degenerate Herman rings" studied by R. Pérez-Marco (in particular, any point of $K$ is recurrent). In any other case (that is, if this number is rational or if $\mathbb{C} \backslash K$ has more than two connected components), the situation is essentially trivial: the dynamics is of Morse-Smale type, and a complete description and classification modulo analytic conjugacy is given.
\end{abstract}

\section{Introduction}

1. Notons $\mathcal{K}$ l'ensemble des paires $(K, F)$ telles que :

- $K$ est un sous-ensemble compact connexe de $\mathbb{C}$, non réduit à un point.

- $F$ est une application univalente (c'est-à-dire holomorphe et injective) dans un voisinage ouvert de $K$, qui vérifie $F(K)=K$.

L'objectif principal de ce travail est de donner une classification des différents types de comportement dynamique que peut avoir $F_{\mid K}$ lorsque $(K, F) \in \mathcal{K}$.

Des exemples classiques de tels objets sont :

- Les fleurs de Fatou locales associées à un point fixe indifférent rationnel.

- Les domaines de linéarisation (disques de Siegel) associés à un point fixe indifférent irrationnel linéarisable.

Récemment, R. Pérez-Marco a montré que, si $F$ est une application holomorphe qui admet un point fixe indifférent $z_{0}$, alors il existe toujours au voisinage de $z_{0}$ des compacts connexes invariants par $F$ non réduits

1991 Mathematics Subject Classification: 30D40, 58F23. 
à $z_{0}$. Plus précisément, pour tout voisinage ouvert $V$ simplement connexe de $z_{0}$ tel que $F$ est univalente au voisinage de $\bar{V}$, il existe un compact connexe $K$, inclus dans $\bar{V}$, contenant $z_{0}$, tel que $F(K)=K$ et $K \cap \partial V \neq \emptyset$ ([PM1]). Si le multiplicateur en $z_{0}$ est rationnel, ces compacts sont tout simplement des fleurs de Fatou (locales). S'il est irrationnel, il peut s'agir de disques (fermés) locaux de Siegel, mais $F$ peut aussi ne pas être linéarisable au voisinage de $z_{0}$; dans ce cas, ces compacts présentent une topologie très complexe (d'où leur nom de "hérissons") et non encore complètement élucidée (voir [PM2]). La dynamique de $F$ sur ces compacts est néanmoins aujourd'hui assez bien comprise ([PM3]); elle ressemble à beaucoup d'égards à celle de la rotation irrationnelle dont l'angle correspond au multiplicateur en $z_{0}$; en particulier, pour chacun de ces compacts, il existe une sous-suite des itérés de $F$ qui converge vers l'identité (uniformément) sur ce compact.

Des résultats analogues sont valables pour les difféomorphismes analytiques du cercle. Si $\varphi$ est un difféomorphisme analytique du cercle $\mathbb{T}^{1}=\mathbb{R} / \mathbb{Z}$, prolongé en une application univalente dans un voisinage de $\mathbb{T}^{1}$ dans $\mathbb{C} / \mathbb{Z}$, alors, pour tout anneau ouvert $V$ contenant $\mathbb{R} / \mathbb{Z}$ tel que $\varphi$ soit univalente au voisinage de $\bar{V}$, il existe un compact connexe $K$ contenant $\mathbb{R} / \mathbb{Z}$, inclus dans $\bar{V}$, tel que $F(K)=K$ et tel que $K$ rencontre les deux composantes connexes de $\partial V$. Notons $\varrho$ le nombre de rotation de $\varphi$. Si $\varrho$ est rationnel, ces compacts ressemblent à des fleurs de Fatou contenant $\mathbb{R} / \mathbb{Z}$. Si $\varrho$ est irrationnel, ils peuvent être des anneaux fermés, mais, si $\varphi$ n'est pas linéarisable, ils présentent là encore une topologie très complexe, alors que la dynamique de $\varphi$ sur ces compacts garde beaucoup de propriétés de la rotation d'angle $\varrho$.

Tous ces résultats ont fortement motivé le présent travail; l'objectif en est d'étudier systématiquement la dynamique des applications $F_{\mid K}$ lorsque $(K, F)$ appartient à $\mathcal{K}$. Nous montrerons en particulier que seulement deux types de dynamiques sont possibles : une dynamique de type Morse-Smale (comme dans le cas des fleurs de Fatou) ou une dynamique qui ressemble à celle d'une rotation irrationnelle (comme dans le cas des disques de Siegel et des hérissons).

2. Considérons une paire $(K, F) \in \mathcal{K}$, et notons $V$ un ouvert connexe contenant $K$ sur lequel $F$ est définie et univalente. Le nombre de composantes connexes de $\mathbb{C} \backslash K$ est bien entendu quelconque, et peut être infini. Lorsque ce nombre vaut 1 ou 2, nous allons définir un nombre de rotation naturel associé à la dynamique.

Supposons tout d'abord que $\mathbb{C} \backslash K$ soit connexe (on dira que $K$ est plein), et considérons une représentation conforme $h: \overline{\mathbb{C}} \backslash \overline{\mathbb{D}} \rightarrow \overline{\mathbb{C}} \backslash K$ ( $(\overline{\mathbb{D}}$ désigne le disque unité fermé). L'application $h^{-1} \circ F \circ h$ se prolonge par réflexion 
de Schwarz en une application univalente au voisinage de $\partial \mathbb{D}=\mathbb{S}^{1}$. La restriction de cette application à $\mathbb{S}^{1}$ définit un difféomorphisme analytique du cercle dont le nombre de rotation (dans $\mathbb{R} / \mathbb{Z}$ ) ne dépend pas du choix de $h$; on l'appellera nombre de rotation associé à $K$.

Supposons maintenant que $\overline{\mathbb{C}} \backslash K$ ait deux composantes connexes (on dira que $K$ est annulaire); notons-les $U_{\text {int }}$ et $U_{\text {ext }}$ ( $U_{\text {int }}$ désigne celle qui est bornée). Supposons en outre que $F\left(U_{\text {int }} \cap V\right) \subset U_{\text {int }}$ et $F\left(U_{\text {ext }} \cap V\right) \subset U_{\text {ext }}$ (cette propriété est toujours vérifiée par l'application $F \circ F$ ). Considérons deux représentations conformes $h_{\text {int }}: \mathbb{D} \rightarrow U_{\text {int }}$ et $h_{\text {ext }}: \overline{\mathbb{C}} \backslash \overline{\mathbb{D}} \rightarrow U_{\text {ext }}$. Les applications $h_{\text {int }}^{-1} \circ F \circ h_{\text {int }}$ et $h_{\text {ext }}^{-1} \circ F \circ h_{\text {ext }}$ se prolongent encore en deux difféomorphismes analytiques du cercle dont les nombres de rotation ne dépendent pas du choix de $h_{\text {int }}$ et $h_{\text {ext }}$. Nous montrerons la

Proposition 1. Ces deux nombres de rotation coïncident.

On pourra donc encore parler de nombre de rotation associé à $K$ lorsque $K$ est annulaire.

Remarque. Cet énoncé est faux en général si l'application $F$ n'est pas holomorphe (par exemple seulement de classe $C^{\infty}$ ou même $\mathbb{R}$-analytique; voir $[\mathrm{L}],[\mathrm{B}])$.

Pour $(K, F) \in \mathcal{K}$ quelconque, introduisons la

Définition 1. On dira que la dynamique de F sur K est de type MorseSmale si les trois assertions suivantes sont vérifiées :

(i) $F_{\mid K}$ a un nombre fini de points périodiques.

(ii) Les ensembles $\alpha$-limite et $\omega$-limite de tout point de $K$ sont deux orbites périodiques.

(iii) Toute orbite périodique indifférente de $F_{\mid K}$ a un multiplicateur rationnel.

Remarque. (1) Dans la définition ci-dessus, l'assertion (iii) est en fait, comme nous le verrons, une conséquence des assertions (i) et (ii) (on peut donc l'omettre).

(2) Cette définition autorise l'existence de points périodiques "paraboliques" (i.e. de multiplicateur indifférent rationnel). Néanmoins, la dynamique au voisinage de ces orbites (dynamique de la fleur de Fatou) a un caractère nettement "hyperbolique" (pétales attractifs, répulsifs, directions stables et instables, etc.); la définition adoptée ci-dessus est donc la plus naturelle dans notre cadre.

L'objectif principal de ce travail est d'établir le résultat de classification suivant : 
ThÉORÈme 1. Soit $(K, F) \in \mathcal{K}$ quelconque. On est toujours dans un (et un seul) des quatre cas suivants:

(a) $K$ est plein et de nombre de rotation irrationnel.

(b) $K$ est annulaire et de nombre de rotation irrationnel.

(c) La dynamique de $F$ sur $K$ est de type Morse-Smale.

(d) $F_{\mid K}$ est racine de l'identité (c'est-à-dire qu'il existe $n \in \mathbb{N}$ tel que $\left.F_{\mid K}^{n}=\mathrm{Id}\right)$.

En particulier, on a le

Corollaire 1. Supposons que $F_{\mid K}$ ne soit pas racine de l'identité et que le nombre de composantes connexes de $\overline{\mathbb{C}} \backslash K$ soit supérieur ou égal à 3 . Alors la dynamique de $F$ sur $K$ est de type Morse-Smale.

L'intérêt et la nouveauté de ces résultats résident dans le fait que tous les compacts connexes de $\mathbb{C}$, et non seulement les compacts connexes pleins, sont considérés. Le cas des compacts connexes pleins était déjà très bien compris grâce aux travaux de Pérez-Marco ([PM1], [PM3]). Tous les résultats énoncés dans cette introduction et concernant les compacts connexes pleins ont d'ailleurs été récemment obtenus de façon indépendante par Pérez-Marco ([PM4]).

Il est naturel de chercher à préciser la topologie de $K$ et la dynamique de $F$ sur $K$ dans chacun des cas (a), (b), et (c) distingués par le théorème ci-dessus (le cas (d) ne présente pas grand intérêt). Considérons tout d'abord le cas où $K$ est plein ou annulaire de nombre de rotation irrationnel. Dans ce cas les techniques introduites par Pérez-Marco pour étudier les compacts connexes pleins ([PM3]) s'adaptent également (grâce à la proposition 1) aux compacts annulaires et permettent de retrouver des résultats très similaires à ceux de [PM3], qui font l'objet du théorème suivant :

ThÉorÈme 2. Soit $(K, F) \in \mathcal{K}$ quelconque. Supposons que $K$ soit plein ou annulaire, et de nombre de rotation $\alpha$ irrationnel. Alors :

(1) Si $K$ est plein, $K$ contient un unique point $z_{0}$ fixe par $F$, de multiplicateur $e^{2 i \pi \alpha}$, et ne contient pas de point périodique autre que $z_{0}$. Si K est annulaire, $K$ ne contient aucun point périodique par $F$.

(2) Les itérés de $F_{\mid K}$ accumulent l'identité (pour la topologie de la convergence uniforme sur $K)$; en particulier, tout point de $K$ est récurrent.

(3) Tout point de $K$ "tourne à la vitesse $\alpha$ " (voir la remarque ci-dessous).

(4) Supposons que int $K$ soit non vide; alors int $K$ est connexe. Si $K$ est plein, int $K$ est simplement connexe et contient $z_{0}$. Si $K$ est annulaire, alors int $K$ est un anneau. Dans les deux cas, $F_{\mid \operatorname{int} K}$ est analytiquement conjuguée à la rotation $z \mapsto e^{2 i \pi \alpha} z$. 
Nous donnerons par ailleurs une démonstration, différente et peut-être plus simple que celle de [PM1], de l'assertion (1) de ce théorème (voir $§ 1.2$ et 1.3$)$.

Remarque. Explicitons ce qu'il faut entendre par "tourner à la vitesse $\alpha$ " dans l'assertion (3) du théorème ci-dessus. Supposons par exemple que $K$ soit plein, et que 0 soit l'unique point fixe de $F$ sur $K$. Considérons un relevé $\widetilde{F}$ de $F$ par le revêtement $E: \mathbb{C} \rightarrow \mathbb{C}^{*}, \zeta \mapsto e^{2 i \pi \zeta}$. Soit $\widetilde{\alpha}$ tel que $\lim _{\operatorname{Im} \zeta \rightarrow+\infty}(\widetilde{F}(\zeta)-\zeta)=\widetilde{\alpha}$; alors $\pi(\widetilde{\alpha})=\alpha$ ( $\pi$ désigne la projection canonique $\mathbb{C} \rightarrow \mathbb{C} / \mathbb{Z}$ ). On dit alors qu'un point $z$ de $K$ "tourne à la vitesse $\alpha$ "si, pour tout point $\zeta \in E^{-1}(\{z\})$, on a

$$
\lim _{n \rightarrow+\infty}\left(\widetilde{F}^{n}(\zeta)-\zeta\right) / n=\widetilde{\alpha}
$$

Si $K$ est annulaire, on suppose que 0 est cette fois un point de la composante connexe bornée de $\mathbb{C} \backslash K$ et on adopte la même convention.

On déduit facilement des théorèmes 1 et 2 le

Corollatre 2. Soient $K_{1}$ et $K_{2}$ deux compacts de $\mathbb{C}$ et $F$ une application univalente au voisinage de $K_{1} \cup K_{2}$ tels que :

(i) Pour tout $i \in\{1,2\},\left(K_{i}, F\right) \in \mathcal{K}$ et $K_{i}$ est plein ou annulaire, de nombre de rotation irrationnel.

(ii) $K_{1} \cap K_{2} \neq \emptyset$.

Alors $K_{1} \cup K_{2}$ est plein ou annulaire et de nombre de rotation irrationnel. De plus, les nombres de rotation de $K_{1}, K_{2}$, et $K_{1} \cup K_{2}$ coïncident.

La topologie des compacts pleins ou annulaires de nombre de rotation irrationnel n'est pas encore complètement comprise, même si on sait qu'elle peut être très complexe ([PM2]). Par exemple, on ne sait pas si un compact annulaire de nombre de rotation irrationnel et d'intérieur vide contient toujours une courbe de Jordan (si une telle courbe de Jordan existe, elle est forcément invariante par la dynamique).

En revanche, nous allons voir maintenant que dans tous les autres cas (c'est-à-dire dès que $K$ n'est pas plein ou annulaire de nombre de rotation irrationnel), la situation est assez simple et peut être complètement décrite.

Considérons une paire $(K, F) \in \mathcal{K}$ quelconque. Supposons tout d'abord que le nombre de composantes connexes de $\overline{\mathbb{C}} \backslash K$ soit fini mais quelconque; notons $n$ ce nombre et notons $U_{1}, \ldots, U_{n}$ ces composantes. Supposons en outre que, pour tout $i \in\{1, \ldots, n\}$, on ait $F\left(U_{i} \cap V\right) \subset U_{i}$ (cette hypothèse est toujours vérifiée quitte à remplacer $F$ par un de ses itérés). A chacune de ces composantes peut alors être associé un nombre de rotation (comme on l'a fait pour les compacts pleins ou annulaires). Nous montrerons la

Proposition 2. Si $n \geq 3$, ces nombres de rotation sont tous nuls. 
De plus, quitte à remplacer $K$ par un compact invariant un peu plus gros, nous allons voir qu'il s'agit d'un objet dont la topologie est très simple. Introduisons la

DÉFinition 2. On appellera pétale invariant par $F$ tout ensemble $P$ ouvert, connexe, simplement connexe, inclus et relativement compact dans $\mathbb{C}$, satisfaisant aux propriétés suivantes :

(1) $\bar{P} \subset V, F(P)=P$, et $F_{\mid P}$ est sans point fixe.

(2) Il existe deux points $x_{\alpha}$ et $x_{\omega}$ de $\partial P$, fixes par $F$, tels que, pour tout $z \in P$, on ait $\lim _{k \rightarrow+\infty} F^{k}(z)=x_{\omega}$ et $\lim _{k \rightarrow-\infty} F^{k}(z)=x_{\alpha}$.

Remarque. Nous verrons que la propriété (2) ci-dessus est en fait une conséquence de la propriété (1).

Nous montrerons le

THÉORÈme 3. Si $n=1$ ou 2 , notons $p / q(p \wedge q=1, q>0)$ le nombre de rotation associé à $K$; si $n \geq 3$, posons $q=1$. Alors :

(1) Tout point $z$ de $K$ périodique par $F$ est accessible par le complémentaire de $K$ et vérifie $F^{q}(z)=z$ et $\left|F^{\prime q}(z)\right| \neq 1$ ou $F^{\prime q}(z)=1$.

(2) Il existe un compact $\widehat{K}$ vérifiant les propriétés suivantes:

(i) $K \subset \widehat{K} \subset V$,

(ii) $F(\widehat{K})=\widehat{K}$

(iii) int $\widehat{K}$ est la réunion d'un nombre fini de pétales invariants par $F^{q}$, deux à deux disjoints.

(iv) $K \backslash \operatorname{Per} F \subset \operatorname{int} \widehat{K}$ (Per $F$ désigne l'ensemble des points périodiques par $F$ ).

REMARQUE. Nous verrons que ce théorème est valable avec une définition plus restrictive de la notion de pétale, pour laquelle tout pétale a un bord analytique par morceaux et est de l'un des trois types dessinés ci-dessous :

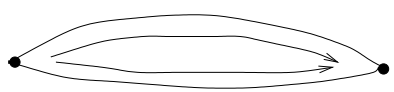

1

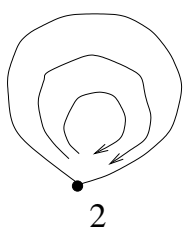

2

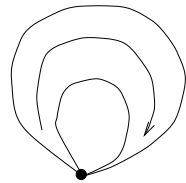

3

Ceci permet une description particulièrement simple de la topologie de $K$ et de la dynamique de $F$ sur $K$; le dessin suivant montre un exemple typique : 


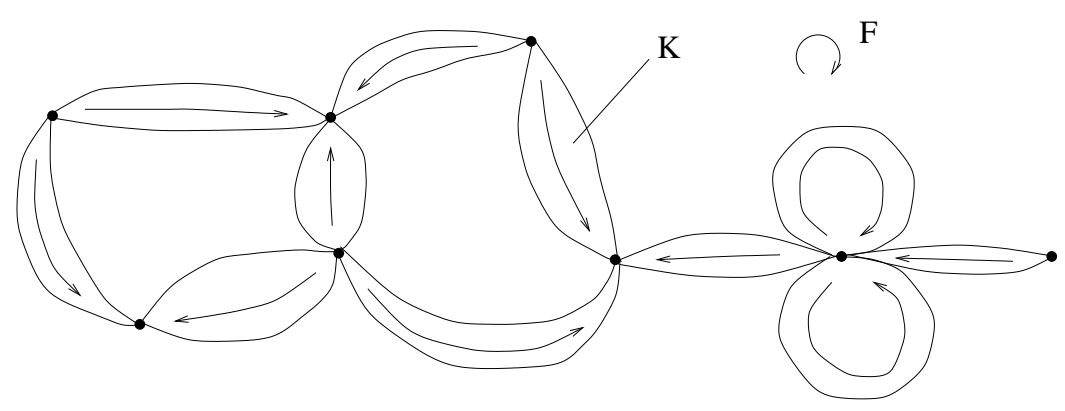

En particulier, la dynamique de $F$ sur $K$ est de type Morse-Smale; ceci démontre le théorème 1 dans le cas particulier où le nombre de composantes connexes de $\mathbb{C} \backslash K$ est fini.

Reste le cas où le nombre de composantes connexes de $\mathbb{C} \backslash K$ est infini. On peut alors se ramener au cas où ce nombre est fini, grâce à la proposition suivante (dont l'idée se trouve dans [H2], et dont nous donnons tout de suite une démonstration) :

Proposition 3. Il existe une paire $(\widehat{K}, \widehat{F})$ appartenant à $\mathcal{K}$, et un homéomorphisme quasi-conforme $\phi: \mathbb{C} \rightarrow \mathbb{C}$ tels que :

(1) $\phi(K) \subset \widehat{K}, \widehat{F}(\phi(K))=\phi(K)$ et $\widehat{F} \circ \phi(z)=\phi \circ F(z)$ pour tout $z \in K$.

(2) $\mathbb{C} \backslash \widehat{K}$ a un nombre fini de composantes connexes.

Dém o nstration. Supposons que le nombre de composantes connexes de $\overline{\mathbb{C}} \backslash K$ soit infini. Notons $U_{i}, i \in I$, ces composantes connexes (indexées par un ensemble $I$ infini). Soit $V$ un ouvert connexe de $\mathbb{C}$ contenant $K$ sur lequel $F$ est univalente.

Remarquons tout d'abord que seulement un nombre fini de composantes connexes de $\overline{\mathbb{C}} \backslash K$ rencontrent $\overline{\mathbb{C}} \backslash V$ (en effet, les composantes connexes de $\overline{\mathbb{C}} \backslash K$ forment un recouvrement du compact $\overline{\mathbb{C}} \backslash V$ par des ouverts disjoints). Notons $I_{1}=\left\{i \in I \mid U_{i} \not \subset V\right\}$ ( $I_{1}$ est fini). Soit $F_{1}$ une extension $\kappa$-quasiconforme, $\kappa<1$, de $F$, c'est-à-dire un homéomorphisme $\kappa$-quasi-conforme de $\overline{\mathbb{C}}$ qui coïncide avec $F$ sur un voisinage $W$ de $K \cup \bigcup_{i \in I \backslash I_{1}} U_{i}$ (il est clair qu'une telle extension existe : n'importe quel difféomorphisme de classe $C^{1}$ de $\overline{\mathbb{C}}$ qui coïncide avec $F$ sur un voisinage de $K \cup \bigcup_{i \in I \backslash I_{1}} U_{i}$ convient).

Posons

$$
\mathcal{U}=\overline{\mathbb{C}} \backslash K, \quad \mathcal{U}_{1}=\bigcup_{i \in I_{1}, k \in \mathbb{Z}} F_{1}^{k}\left(U_{i}\right), \quad \mathcal{U}_{2}=\mathcal{U} \backslash \mathcal{U}_{1} .
$$

Soit $i \in I$; on dira que $U_{i}$ est périodique s'il existe $k \in \mathbb{N}$ tel que $F_{1}^{k}\left(U_{i}\right)=$ $U_{i}$. Notons $\mathcal{U}_{1 \text {,per }}$ la réunion des composantes connexes de $\mathcal{U}_{1}$ qui sont périodiques sous l'action de $F_{1}$, et notons $\mathcal{U}_{1 \text {,err }}=\mathcal{U} \backslash \mathcal{U}_{1 \text {,per }}$ (ce sont les 
composantes connexes errantes). On a

$$
\overline{\mathbb{C}}=K \cup \mathcal{U}_{1, \text { per }} \cup \mathcal{U}_{1, \text { err }} \cup \mathcal{U}_{2}
$$

et ces ensembles sont tous invariants par $F_{1}$.

Posons $K_{1}=K \cup \mathcal{U}_{1, \text { err }} \cup \mathcal{U}_{2}$. Construisons une forme de Beltrami $\mu \in$ $L^{\infty}(\overline{\mathbb{C}})$ avec les caractéristiques suivantes :

- Sur $K \cup \mathcal{U}_{1, \text { per }} \cup \mathcal{U}_{2}, \mu=0$.

- Sur $\mathcal{U}_{1, \text { err }}, \mu$ est $F_{1}$-invariante quelconque, d'ellipticité bornée.

Pour construire une telle forme $\mu$ sur $\mathcal{U}_{1 \text {,err }}$, il suffit, pour chaque orbite de composantes connexes de $\mathcal{U}_{1 \text {,err }}$, de poser $\mu=0$ sur une des composantes de l'orbite, puis de transporter $\mu$ par $F_{1}$. La forme est alors $F_{1}$-invariante (par construction) et l'ellipticité est bornée car seul un nombre fini de composantes connexes de l'orbite ne sont pas incluses dans $W$, c'est-à-dire contiennent des points où $F_{1}$ n'est pas holomorphe.

La forme obtenue est invariante par $F_{1}$ au voisinage de $K_{1}$. Soit $\phi$ un homéomorphisme quasi-conforme de $\overline{\mathbb{C}}$ qui vérifie $\phi_{*} \mu=\mu_{0}$ ( $\mu_{0}$ désigne la structure complexe standard sur $\overline{\mathbb{C}}$ ). Posons

$$
\widehat{F}=\phi \circ F_{1} \circ \phi^{-1}, \quad \widehat{K}=\phi\left(K_{1}\right) .
$$

L'application $\widehat{F}$ est alors univalente au voisinage de $\widehat{K}$, et vérifie $\widehat{F}(\widehat{K})=\widehat{K}$. De plus, le nombre de composantes connexes de $\overline{\mathbb{C}} \backslash \widehat{K}$ est fini.

La proposition 3 est donc démontrée.

On en déduit, grâce au théorème 3 ci-dessus, que, si $(K, F)$ est une paire appartenant à $\mathcal{K}$ quelconque, et si $K$ n'est pas plein ou annulaire de nombre de rotation irrationnel, alors la dynamique de $F$ sur $K$ est de type MorseSmale (c'est le théorème 1) et il existe un entier $q$ tel que les conclusions (1) et (2) du théorème 3 soient valables.

Finalement, considérons une paire $(K, F) \in \mathcal{K}$ quelconque telle que $K$ soit plein ou annulaire de nombre de rotation rationnel; nous montrerons la

Proposition 4. (1) Supposons que $K$ soit plein et de nombre de rotation rationnel $p / q, p \wedge q=1, q>0$. Alors il existe un point fixe sur $K$ qui est soit non indifférent, soit de multiplicateur $e^{2 i \pi p / q}$. Tout autre point périodique de $K$ a pour période minimale $q$. De plus, si le point fixe a pour multiplicateur $e^{2 i \pi p / q}$, alors tout point de $K$ "tourne à la vitesse $p / q$ ".

(2) Supposons que $K$ soit annulaire et de nombre de rotation $p / q$. Alors tout point périodique de $K$ a pour période minimale q, et tout point de $K$ "tourne à la vitesse $p / q "$. De plus, pour tout voisinage ouvert $V$ de $K$, il existe une courbe de Jordan $J$, incluse dans $V$, invariante par $F$, et non disjointe de $K$; si $V$ est un anneau ouvert tel que $K$ sépare les deux composantes connexes de $\partial V$, alors $J$ les sépare également. D'autre part, on peut demander que $J$ ne rencontre $K$ qu'en des points périodiques par $F$. 
Signalons que l'ensemble de ces résultats entraîne immédiatement le résultat principal de [H2], à savoir :

Si $R$ est une fraction rationnelle sur $\overline{\mathbb{C}}$ qui admet un domaine de rotation $\mathcal{D}$ (disque de Siegel ou anneau de Herman) et que le nombre de rotation associé à ce domaine satisfait à la condition arithmétique $\mathcal{H}$ de linéarisation analytique globale des difféomorphismes analytiques du cercle (voir [H1] et $[\mathrm{Y}]$ ), alors $R$ n'est pas injective au voisinage de $\overline{\mathcal{D}}(\mathcal{D}$ est supposé maximal).

Le présent travail offre donc une nouvelle démonstration de ce résultat, une variante qui n'utilise pas le théorème du noyau de Carathéodory (ce dernier est un outil essentiel dans la preuve de [H2], et aussi dans certains résultats de [PM1] redémontrés ici d'une manière différente).

3. Décrivons maintenant rapidement l'organisation de cet article.

La première partie est consacrée, après quelques résultats préparatoires $(\S 1.1)$, à la démonstration des résultats techniques principaux, à savoir la proposition $1(\S 1.2)$ et la proposition $2(\S 1.4)$. Les démonstrations sont basées sur un argument d'intégrale de Cauchy, dont l'idée est très simple : il ne peut pas y avoir de "cisaillement" au voisinage d'un compact connexe invariant; elles utilisent de manière essentielle un lemme sur les difféomorphismes analytiques du cercle (lemme 1.1.3) démontré dans [H1]. La même méthode permet de démontrer assez simplement l'assertion (1) du théorème 2 ( $\$ 1.2$ et 1.3$)$. Enfin, on montre dans le paragraphe 1.5 comment ces résultats permettent d'établir les autres assertions du théorème 2 (la démarche est semblable à celle de [PM3]).

La deuxième partie est consacrée à l'étude des compacts connexes invariants qui ne sont pas pleins ou annulaires de nombre de rotation irrationnel. Dans le paragraphe 2.1 , on démontre le théorème 3 , c'est-à-dire la décomposition de $K$ en pétales périodiques (la proposition 2 est essentielle pour cette démonstration). On en déduit le théorème 1 dans le cas particulier où le nombre de composantes connexes de $\mathbb{C} \backslash K$ est fini, et on montre que la proposition 3 entraîne ce théorème dans le cas général. Le paragraphe 2.2 est consacré à la démonstration de la proposition 4 .

En appendice, on définit la notion de plongement analytique de type Morse-Smale de surfaces de Riemann (cette notion englobe en particulier les applications $F$ univalentes au voisinage d'un compact $K$ lorsque $(K, F) \in \mathcal{K}$ et que la dynamique de $F$ sur $K$ est de type Morse-Smale au sens de la définition 1). On montre que ces plongements peuvent être assez simplement classifiés modulo conjugaison analytique. Cette classification fait apparaître des invariants locaux (multiplicateurs aux points fixes et, lorsque ces points fixes sont indifférents rationnels, invariants de conjugaison d'ÉcalleVoronin) et des invariants de recollement (invariants de Mather) entre ces objets locaux. La formulation adoptée est proche de celle de Voronin ([V]). 
4. Ce travail constitue une partie de ma thèse, effectuée sous la direction de Jean-Christophe Yoccoz, et n'aurait pas vu le jour sans les idées, les conseils, et les encouragements qu'il m'a toujours prodigués. En particulier, je le remercie de m'avoir montré comment modifier et simplifier considérablement la démonstration de la proposition 1 (qui est un point essentiel), ainsi que de m'avoir aidé à améliorer la présentation générale. Je tiens également à remercier Ricardo Pérez-Marco : ses récents travaux sur les disques de Siegel dégénérés ([PM3]), auxquels j'ai eu accès pratiquement "en temps réel", ont exercé une influence considérable sur ce travail. En outre, il a obtenu indépendamment une partie des résultats présentés ici, plus précisément les résultats concernant les compacts pleins (voir [PM4]). Je remercie Alexis Marin pour ses réponses diligentes et compétentes à mes questions concernant les problèmes de topologie plane. Enfin, je remercie le "referee" pour m'avoir indiqué comment simplifier considérablement la démonstration de la proposition 1.4.1.

5. Notations. On note $\mathbb{T}^{1}=\mathbb{R} / \mathbb{Z}$ et $\overline{\mathbb{C}} / \mathbb{Z}$ la surface de Riemann difféomorphe à $\overline{\mathbb{C}}$ obtenue en rajoutant deux points, $+i \infty$ et $-i \infty$, aux deux bouts de $\mathbb{C} / \mathbb{Z}$. On notera toujours $\pi$ la projection canonique $\mathbb{C} \rightarrow \mathbb{C} / \mathbb{Z}$.

On note $\mathbb{H}^{*}=\{z \in \mathbb{C} \mid \operatorname{Im} z<0\}$ et, pour tout $\Delta>0, \widetilde{A}_{\Delta}=\{z \in \mathbb{C} \mid$ $|\operatorname{Im} z|<\Delta\}$ et $A_{\Delta}=\pi\left(\widetilde{A}_{\Delta}\right)$.

Enfin, on note Diff ${ }^{\omega}\left(\mathbb{T}^{1}\right)$ (resp. Diff ${ }^{\omega}\left(\mathbb{S}^{1}\right)$ ) les difféomorphismes analytiques du cercle $\mathbb{T}^{1}$ (resp. $\mathbb{S}^{1}$ ) préservant l'orientation, et $D^{\omega}\left(\mathbb{T}^{1}\right)$ l'ensemble des relevés à $\mathbb{R}$ des éléments de $\operatorname{Diff}{ }^{\omega}\left(\mathbb{T}^{1}\right)$.

\section{Coïncidence des nombres de rotation}

\subsection{Préliminaires}

Lemme 1.1.1 (voir par exemple $[\mathrm{C}, \mathrm{G}]$, p. 77 ). Soit $W$ un ouvert connexe de $\mathbb{C}$, dont le complémentaire dans $\mathbb{C}$ n'est pas réduit à un point, et $F$ une application univalente au voisinage de l'adhérence de $W$ dans $\overline{\mathbb{C}}$, vérifiant $F(W) \subset W$. Supposons qu'il existe $z_{0} \in W$ tel que $F^{n}\left(z_{0}\right) \rightarrow \partial W$ lorsque $n \rightarrow+\infty$. Alors :

(i) Il existe $z_{1} \in \partial W$ fixe par $F$ tel que, pour tout $z \in W, F^{n}(z) \rightarrow z_{1}$ lorsque $n \rightarrow+\infty$.

(ii) $\left|F^{\prime}\left(z_{1}\right)\right| \neq 1$ ou $F^{\prime}\left(z_{1}\right)=1$.

Démonstration. L'assertion (i) se démontre facilement en utilisant la métrique de Poincaré de $W$, qui explose au bord, et pour laquelle $F_{\mid W}$ est une isométrie. L'assertion (ii) est une conséquence du Snail lemma.

Considérons une paire $(U, F)$ satisfaisant aux hypothèses suivantes : 
(i) $U$ est un ouvert de $\overline{\mathbb{C}}$, connexe, simplement connexe, et $\mathbb{C} \backslash U$ n'est pas vide ni réduit à un point.

(ii) $F$ est une application définie et univalente sur un voisinage ouvert $V$ de $\partial U$, à valeurs dans $\overline{\mathbb{C}}$, et vérifiant $F(\partial U)=\partial U$ et $F(U \cap V) \subset U(\partial U$ désigne le bord de $U$ dans $\overline{\mathbb{C}}$ ).

Considérons une représentation conforme $h: \mathbb{D} \rightarrow U$; l'application $h^{-1} \circ$ $F \circ h$ se prolonge alors par réflexion de Schwarz en un difféomorphisme analytique du cercle $\mathbb{S}^{1}=\partial \mathbb{D}$, préservant l'orientation; notons-le $\varphi$, et notons $\varrho(\varphi)$ son nombre de rotation (il ne dépend que de $(U, F)$ ).

Nous allons effectuer deux constructions différentes, selon que $\varrho(\varphi)$ est rationnel ou irrationnel, qui vont être utilisées plusieurs fois par la suite.

(a) Construction lorsque le nombre de rotation est rationnel. Supposons que $\varrho(\varphi)$ soit rationnel, et que $F_{\mid \partial U}$ ne soit pas une racine de l'identité. Notons $p / q \in \mathbb{Q}, p \wedge q=1, q>0$, un relevé de $\varrho(\varphi)$ dans $\mathbb{Q}$.

Puisque $F_{\mid \partial U}$ n'est pas racine de l'identité, $\varphi$ n'est pas linéarisable. Il existe donc $k \in \mathbb{N}^{*}$ tel que $\varphi$ admette exactement $k q$ points périodiques. Notons $\left(y_{i}\right)_{i \in \mathbb{Z} / k q \mathbb{Z}}$ le $k q$-uplet formé de ces points périodiques ordonnés dans l'ordre cyclique de $\mathbb{S}^{1}$. Pour tout $i \in \mathbb{Z} / k q \mathbb{Z}$, on a $\varphi\left(y_{i}\right)=y_{i+p k}$.

Notre construction est résumée par le lemme suivant :

LEMME 1.1.2. (1) La représentation conforme $h: \mathbb{D} \rightarrow U$ admet une limite non tangentielle (voir $[\mathrm{C}, \mathrm{G}]$ ou $[\mathrm{Po}]$ ) en chacun des points $y_{i}, i \in$ $\mathbb{Z} / k q \mathbb{Z}$ (pour tout $i \in \mathbb{Z} / k q \mathbb{Z}$, notons $x_{i} \in \partial U$ la limite non tangentielle de $h$ en $\left.y_{i}\right)$.

(2) Il existe un $k q$-uplet $\left(\gamma_{i}\right)_{i \in \mathbb{Z} / k q \mathbb{Z}}$ de chemins $\mathbb{R} \rightarrow U$, analytiques, injectifs, d'images deux à deux disjointes, et un $k q$-uplet $\left(\sigma_{i}\right)_{i \in \mathbb{Z} / k q \mathbb{Z}}$ d'éléments de $\{-1,1\}$, tels que, pour tout $i \in \mathbb{Z} / k q \mathbb{Z}$, on ait :

(i) $F\left(x_{i}\right)=x_{i+p k}$ et $F \circ \gamma_{i}(\mathbb{R})=\gamma_{i+p k}(\mathbb{R})$. En particulier, $F^{q}\left(x_{i}\right)=$ $x_{i}$ et $F^{q} \circ \gamma_{i}(\mathbb{R})=\gamma_{i}(\mathbb{R})$.

(ii) $\left|\left(F^{q}\right)^{\prime}\left(x_{i}\right)\right| \neq 1$ ou $\left(F^{q}\right)^{\prime}\left(x_{i}\right)=1$.

(iii) $\lim _{\sigma_{i} t \rightarrow-\infty} \gamma_{i}(t)=x_{i}$ et $\lim _{\sigma_{i} t \rightarrow+\infty} \gamma_{i}(t)=x_{i+1}$.

(iv) $F^{q} \circ \gamma_{i}(t)=\gamma_{i}(t+1)$. En particulier, tous les points de $\gamma_{i}(\mathbb{R})$ ont sous l'action de $F^{q}$ une orbite hétérocline entre $x_{i}$ et $x_{i}+1$.

L'ensemble $\bigcup_{i \in \mathbb{Z} / k q \mathbb{Z}}\left(\left\{x_{i}\right\} \cup \gamma_{i}(\mathbb{R})\right)$ définit donc une courbe, notée $\mathcal{C}$, fermée, non nécessairement simple, invariante par $F$.

(3) Le complémentaire de $\mathcal{C}$ dans $\overline{\mathbb{C}}$ a une unique composante connexe $\Omega$ entièrement incluse dans $U$ et on a $\mathcal{C}=\partial \Omega$. De plus, on peut choisir les $\gamma_{i}$ pour que $\mathcal{C}$ soit incluse dans un voisinage arbitrairement petit de $\partial U$, plus précisément, pour que $\Omega$ contienne n'importe quel compact de $U$ fixé à l'avance. 


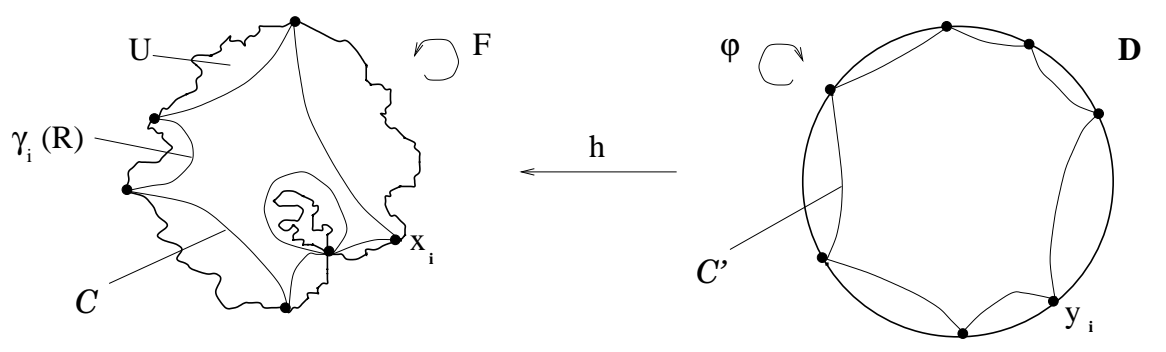

Démonstration. Pour tous $y, y^{\prime}$ appartenant à $\mathbb{S}^{1}$, notons $] y ; y^{\prime}[$ l'intervalle de $\mathbb{S}^{1}$ formé des points strictement compris entre $y$ et $y^{\prime}$ pour l'ordre cyclique de $\mathbb{S}^{1}$.

Fixons $i \in \mathbb{Z} / k q \mathbb{Z}$ quelconque. Alors $\varphi_{\mid] y_{i} ; y_{i+1}[}^{q}$ définit un homéomorphisme sans point fixe de l'intervalle ouvert $] y_{i} ; y_{i+1}\left[\right.$. Posons $\sigma_{i}=+1$ ou -1 selon que $\varphi^{q}-\mathrm{Id}$ est à valeurs positives ou négatives sur $] y_{i} ; y_{i+1}[$. Alors, pour tout $z \in] y_{i} ; y_{i+1}[$, on a

$$
\left(\varphi^{\sigma_{i} q}\right)^{n}(z) \underset{n \rightarrow+\infty}{\longrightarrow} y_{i}, \quad\left(\varphi^{\sigma_{i} q}\right)^{n}(z) \underset{n \rightarrow+\infty}{\longrightarrow} y_{i+1} .
$$

Notons $\left(\right.$ Hét $\left._{i}\right)$ cette propriété d'être hétérocline entre $y_{i}$ et $y_{i+1}$ pour $z$. Il s'agit d'une propriété ouverte, et on montre facilement qu'il existe un domaine de Jordan ouvert $W_{i} \subset \mathbb{D}$, complètement invariant par $\varphi^{q}$, tel que tous les points de $W_{i}$ vérifient (Hét ${ }_{i}$ ) et tel que $] y_{i} ; y_{i+1}\left[\subset \partial W_{i}\right.$. De plus, on peut supposer que $W_{i}$ est inclus dans un voisinage arbitrairement petit de $\mathbb{S}^{1}$.

La variété obtenue en quotientant $W_{i}$ par l'action de $\varphi^{q}$ est une surface de Riemann ouverte, plus précisément un anneau de module fini $m$. Notons $A_{m / 2}=\{z \in \mathbb{C} / \mathbb{Z}|| \operatorname{Im} z \mid<m / 2\}$. Cet ensemble est lui aussi un anneau de module $m$, et d'après le théorème d'uniformisation de Koebe, il existe un difféomorphisme analytique entre ces deux anneaux. L'image de $\mathbb{R} / \mathbb{Z} \subset$ $A_{m / 2}$ par un tel difféomorphisme est une courbe analytique fermée dont l'image réciproque dans $W_{i}$ est un chemin analytique injectif ; on peut choisir une paramétrisation $\mu_{i}: \mathbb{R} \rightarrow W_{i}$ de ce chemin telle que, pour tout $t \in \mathbb{R}$, on ait $\varphi^{q}\left(\mu_{i}(t)\right)=\mu_{i}(t+1)$. Il est clair que $\mu_{i}(t) \rightarrow y_{i}$ lorsque $\sigma_{i} t \rightarrow-\infty$, et $\mu_{i}(t) \rightarrow y_{i+1}$ lorsque $\sigma_{i} t \rightarrow+\infty$.

Posons $V_{i}=h\left(W_{i}\right)$ et $\gamma_{i}=h \circ \mu_{i}$; on a $F^{q} \circ \gamma_{i}(t)=\gamma_{i}(t+1)$, et $V_{i}$ est un domaine ouvert connexe simplement connexe complètement invariant par $F^{q}$. Donc, d'après le lemme 1.1.1, il existe $\alpha_{i}$ et $\omega_{i}$, deux points fixes pour $F^{q}$ appartenant au bord de $V_{i}$ tels que, pour tout $z \in V_{i}$, on ait

$$
\left(F^{q}\right)^{n}(z) \underset{n \rightarrow-\infty}{\longrightarrow} \alpha_{i}, \quad\left(F^{q}\right)^{n}(z) \underset{n \rightarrow+\infty}{\longrightarrow} \omega_{i} .
$$

Cette convergence est uniforme sur tout compact de $V_{i}$, en particulier sur 
$\gamma_{i}([0 ; 1])$, ce qui montre que

$$
\gamma_{i}(t) \underset{t \rightarrow-\infty}{\longrightarrow} \alpha_{i}, \quad \gamma_{i}(t) \underset{t \rightarrow+\infty}{\longrightarrow} \omega_{i} .
$$

L'application $h$ admet donc des limites non tangentielles en chacun des points $y_{i}, i \in \mathbb{Z} / k q \mathbb{Z}$.

Pour tout $i \in \mathbb{Z} / k q \mathbb{Z}$, notons $x_{i}$ la limite non tangentielle de $h$ en $y_{i}$. On vérifie alors que

$$
\gamma_{i}(t) \underset{\sigma_{i} t \rightarrow-\infty}{\longrightarrow} x_{i}, \quad \gamma_{i}(t) \underset{\sigma_{i} t \rightarrow+\infty}{\longrightarrow} x_{i+1} .
$$

En outre, on a clairement $F\left(x_{i}\right)=x_{i+k p}$ et, d'après le lemme 1.1.1, $\left|\left(F^{q}\right)^{\prime}\left(x_{i}\right)\right|$ $\neq 1$ ou $\left(F^{q}\right)^{\prime}\left(x_{i}\right)=1$.

On construit ainsi, lorsque $i$ parcourt $\mathbb{Z} / k q \mathbb{Z}, k q$ ouverts $V_{i}, k q$ chemins analytiques $\gamma_{i}$ et $k q$ couples de points fixes $\left(x_{i}, x_{i}^{\prime}\right)$. Il est clair que l'on peut supposer que $V_{i+k p}=F\left(V_{i}\right)$ et $\gamma_{i+k p}(\mathbb{R})=F \circ \gamma_{i}(\mathbb{R})$. Les assertions (1) et (2) du lemme sont donc démontrées.

Considérons les ensembles

$$
\mathcal{C}=\bigcup_{i \in \mathbb{Z} / k q \mathbb{Z}}\left(\left\{x_{i}\right\} \cup \gamma_{i}(\mathbb{R})\right), \quad \mathcal{C}^{\prime}=\bigcup_{i \in \mathbb{Z} / k q \mathbb{Z}}\left(\left\{y_{i}\right\} \cup \mu_{i}(\mathbb{R})\right) .
$$

L'ensemble $\mathcal{C}^{\prime}$ est une courbe de Jordan incluse dans $\overline{\mathbb{D}}$. Soit $\Omega^{\prime}$ la composante connexe bornée du complémentaire de $\mathcal{C}^{\prime}$ dans $\overline{\mathbb{C}}$. Posons $\Omega=h\left(\Omega^{\prime}\right)$; alors $\Omega \subset U$ et $\mathcal{C}=\partial \Omega$. L'ouvert $\Omega$ est donc une composante connexe du complémentaire de $\mathcal{C}$ dans $\overline{\mathbb{C}}$.

Considérons une composante connexe $X$ de $\overline{\mathbb{C}} \backslash \mathcal{C}$ différente de $\Omega$. Alors $X$ rencontre forcément ${ }^{c} U$. En effet, raisonnons par l'absurde et supposons que $X$ soit toute entière incluse dans $U$. Alors $h^{-1}(X)=Y$ est une composante connexe de $\mathbb{D} \backslash \mathcal{C}^{\prime}$, différente de $\Omega^{\prime}$; le bord de $X$ contient donc un sous-arc ouvert (en particulier de mesure positive) de $\partial \mathbb{D}$. L'ensemble des valeurs limites de $h$ aux points de ce sous-arc est inclus dans $\bar{X} \cap \partial U$. Or, puisque $X \subset U$, on a $\bar{X} \cap \partial U=\partial X \cap \partial U \subset \mathcal{C} \cap \partial U$ qui est fini, ce qui est en contradiction avec le théorème de $\mathrm{F}$. et $\mathrm{M}$. Riesz ([Po]). Finalement, nous avons bien montré que $X$ rencontre le complémentaire de $U$.

Enfin, si $K$ est un compact quelconque de $U, h^{-1}(K)$ est un compact de $\mathbb{D}$, et on peut choisir les chemins $\mu_{i}$ pour que $h^{-1}(K)$ soit inclus dans $\Omega^{\prime}$, d'où $K \subset \Omega$. Ceci termine la démonstration de l'assertion (3).

DÉFInition. Nous appellerons courbe invariante associée à la paire $(U, F)$ toute courbe $\mathcal{C}$ fermée (non nécessairement simple) obtenue à partir de la paire $(U, F)$ par la construction précédente.

(b) Construction lorsque le nombre de rotation est irrationnel. Supposons que $\varrho(\varphi)$ soit irrationnel. Nous allons simplement montrer une construction d'une courbe "quasi-invariante" par $F$ dans un voisinage de $U$.

Nous aurons besoin du résultat suivant, dû à M. R. Herman : 
Lemme 1.1.3 ([H1], prop. 2.3 p. 93). Soit $f \in D^{\omega}\left(\mathbb{T}^{1}\right)$ de nombre de rotation $\alpha$ irrationnel. Alors, pour tout $\varepsilon>0$, il existe $g \in D^{\omega}\left(\mathbb{T}^{1}\right)$ tel que l'application $g \circ f \circ g^{-1}$ soit $\varepsilon$-proche de la translation $t \mapsto t+\alpha$ dans la $C^{1}$-topologie.

Soit $\varepsilon>0$ quelconque; appliquons le lemme précédent à $\varepsilon$ et au difféomorphisme analytique du cercle $\varphi$. Il existe $g \in \operatorname{Diff}^{\omega}\left(\mathbb{S}^{1}\right)$ tel que le difféomorphisme analytique $\psi$ de $\mathbb{S}^{1}$ défini par $\psi=g^{-1} \circ \varphi \circ g$ soit $\varepsilon$-proche de la rotation d'angle $\varrho(\varphi)$ dans la $C^{1}$-topologie.

Pour $\delta>0$, notons $\mathcal{C}_{\delta}$ le cercle de centre 0 et de rayon $e^{-2 \pi \delta}$. La courbe $h \circ g\left(\mathcal{C}_{\delta}\right)$ (bien définie si $\delta$ est assez petit) est ce que nous appellerons une courbe quasi-invariante associée à la paire $(U, F)$; nous aurons à plusieures reprises à considérer de telles courbes.

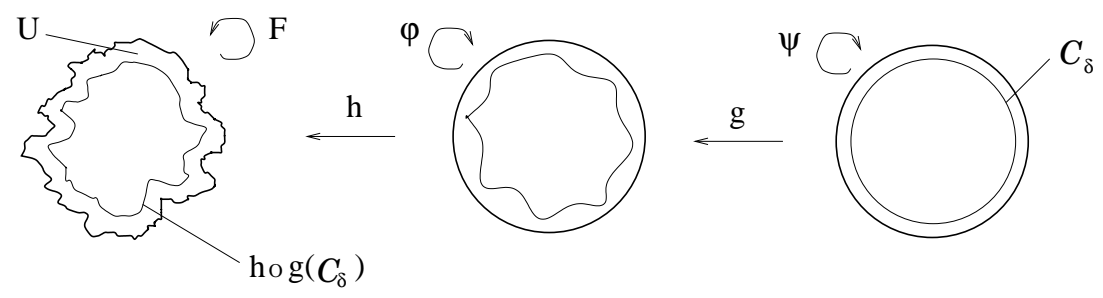

(c) Condition suffisante pour que le nombre de rotation soit rationnel. Reprenons les notations et les hypothèses introduites avant le (a). On a alors le

LEMME 1.1.4. Supposons que $F$ admette sur $\partial U$ un point fixe qui ne soit pas indifférent irrationnel. Alors $\varrho(\varphi) \in \mathbb{Q} / \mathbb{Z}$.

Démonstration. Appelons $z_{0} \in \partial U$ le point fixe de $F$ donné par l'hypothèse.

PREMIER CAS : Supposons que $z_{0}$ ne soit pas indifférent. Alors, quitte à changer $F$ en $F^{-1}$, on peut supposer que $z_{0}$ est attractif.

Soit $\varepsilon>0$ assez petit pour que $F$ soit définie sur $B\left(z_{0}, \varepsilon\right)$, que l'on ait $F\left(B\left(z_{0}, \varepsilon\right)\right) \subset B\left(z_{0}, \varepsilon\right)$, et que les points de $B\left(z_{0}, \varepsilon\right)$ convergent uniformément vers $z_{0}$ sous l'action de $F$. Soit $\eta$ un cross-cut de $U$ d'adhérence incluse dans $B\left(z_{0}, \varepsilon\right) \backslash\left\{z_{0}\right\}$. Alors, pour tout $n \in \mathbb{N}, F^{n}$ est définie sur ce cross-cut, et $F^{n} \circ \eta$ est un cross-cut qui, pour $n$ assez grand, ne rencontre jamais $\eta$. Or, d'après le théorème de F. et M. Riesz et un argument longueur-aire classique (voir par exemple [Po]), $h^{-1}(\eta)$ est également un cross-cut, et d'après la remarque précédente, pour $n$ assez grand, on a $\phi^{n}\left(h^{-1}(\eta)\right) \cap h^{-1}(\eta)=\emptyset$, ce qui est impossible si $\varrho(\varphi) \in(\mathbb{R} \backslash \mathbb{Q}) / \mathbb{Z}$ (d'après le théorème de Denjoy, $\varphi$ est topologiquement conjugué à la rotation d'angle $\alpha$ ). 
DeuxiÈme CAS : Supposons que $z_{0}$ soit indifférent rationnel. Si $z_{0}$ est linéarisable, alors $F_{\mid \partial U}$ est une racine de l'identité, et il est clair que $\varrho\left(F_{\mid U}\right) \in$ $\mathbb{Q} / \mathbb{Z}$. Supposons donc que $z_{0}$ ne soit pas linéarisable.

Dans ce cas, il existe $\varepsilon>0$ tel que $B\left(z_{0}, \varepsilon\right) \backslash\left\{z_{0}\right\}$ soit incluse dans la réunion des pétales attractifs et répulsifs locaux de $z_{0}$ (voir $[\mathrm{C}, \mathrm{G}]$ ). Considérons un point $z_{1} \in\left(B\left(z_{0}, \varepsilon\right) \cap \partial U\right) \backslash\left\{z_{0}\right\}$, et supposons, par exemple, que $z_{1}$ soit inclus dans un pétale attractif de $z_{0}$. Alors il existe un (petit) voisinage ouvert $V$ de $z_{1}$ tel que tous les itérés positifs de $F$ soient définis sur $V$ et tels que les points de $V$ convergent uniformément vers $z_{0}$ sous l'action de $F$. Soit $\eta$ un cross-cut de $U$ tout entier inclus dans $V$. Alors, pour $n$ assez grand, $F^{n}(\eta)$ et $\eta$ sont disjoints, ce qui, encore une fois, est impossible si $\varrho(\varphi) \in(\mathbb{R} \backslash \mathbb{Q}) / \mathbb{Z}$.

1.2. Compact annulaire. Notre premier objectif est de démontrer la proposition 1. Pour simplifier la présentation, on se place dans $\mathbb{C} / \mathbb{Z}$ (qui est plus adapté à la géométrie des compacts annulaires) plutôt que dans $\mathbb{C}$.

Considérons donc un compact connexe $K$, inclus dans $\mathbb{C} / \mathbb{Z}$, annulaire, et une application $F$ univalente dans un voisinage $V$ de $K$, vérifiant $F(K)=K$. Supposons que $K$ sépare les deux bouts de $\mathbb{C} / \mathbb{Z}$, et notons $U_{h}$ et $U_{b}$ les composantes connexes de $\overline{\mathbb{C} / \mathbb{Z}} \backslash K$ contenant respectivement $+i \infty$ et $-i \infty$. Supposons enfin que $F\left(U_{h} \cap V\right) \subset U_{h}$ et $F\left(U_{b} \cap V\right) \subset U_{b}$.

Soient $h_{h}$ et $h_{b}$ des représentations conformes, respectivement de $\mathbb{H} / \mathbb{Z} \cup$ $\{+i \infty\}$ dans $U_{h}$ et de $\mathbb{H}^{*} / \mathbb{Z} \cup\{-i \infty\}$ dans $U_{b}$. Notons $\varphi_{h}$ et $\varphi_{b}$ les difféomorphisme analytique du cercle obtenus en prolongeant respectivement $h_{h}^{-1} \circ F \circ h_{h}$ et $h_{b}^{-1} \circ F \circ h_{b}$ par réflexion de Schwarz. Notons $\alpha_{h}$ et $\alpha_{b}$ les nombres de rotation (dans $\mathbb{R} / \mathbb{Z}$ ) respectivement de $\varphi_{h}$ et de $\varphi_{b}$. Notre objectif est de démontrer que $\alpha_{h}=\alpha_{b}$; nous allons même être un peu plus précis.

Considérons des relevés $\widetilde{F}, \widetilde{h}_{h}$, et $\widetilde{h}_{b}$, respectivement de $F, h_{h}$, et $h_{b}$ par la projection canonique $\pi: \mathbb{C} \rightarrow \mathbb{C} / \mathbb{Z}$. Les applications $\widetilde{\varphi}_{h}$ et $\widetilde{\varphi}_{b}$ obtenues en prolongeant respectivement $\widetilde{h}_{h}^{-1} \circ \widetilde{F} \circ \widetilde{h}_{h}$ et $\widetilde{h}_{b}^{-1} \circ F \circ \widetilde{h}_{b}$ par réflexion de Schwarz sont des relevés respectifs de $\varphi_{h}$ et $\varphi_{b}$. Notons $\widetilde{\alpha}_{h}$ et $\widetilde{\alpha}_{b}$ les nombres de rotation $($ dans $\mathbb{R})$ de $\widetilde{\varphi}_{h}$ et $\widetilde{\varphi}_{b}\left(\right.$ alors $\pi\left(\widetilde{\alpha}_{h}\right)=\alpha_{h}$ et $\left.\pi\left(\widetilde{\alpha}_{b}\right)=\alpha_{b}\right)$. Nous allons en fait montrer la

Proposition 1.2.1. $\widetilde{\alpha}_{h}=\widetilde{\alpha}_{b}$.

Démonstration. Raisonnons par l'absurde et supposons par exemple que $\widetilde{\alpha}_{h}>\widetilde{\alpha}_{b}$. Alors, quitte à remplacer $\widetilde{F}$ par un de ses itérés composé par une translation entière, on peut supposer $\widetilde{\alpha}_{h}>1$ et $\widetilde{\alpha}_{b}<-1$. Distingons trois cas :

PREMIER CAS : $\widetilde{\alpha}_{h}$ et $\widetilde{\alpha}_{b}$ sont tous deux irrationnels. Soit $\varepsilon>0$ un paramètre réel à choisir par la suite. D'après le lemme 1.1.3, il existe deux 
éléments $\widetilde{g}_{h}$ et $\widetilde{g}_{b}$ de $D^{\omega}\left(\mathbb{T}^{1}\right)$ tels que les applications $\widetilde{\psi}_{h}=\widetilde{g}_{h}^{-1} \circ \widetilde{\varphi}_{h} \circ \widetilde{g}_{h}$ et $\widetilde{\psi}_{b}=\widetilde{g}_{b}^{-1} \circ \widetilde{\varphi}_{b} \circ \widetilde{g}_{b}$ vérifient

$$
\left\|\widetilde{\psi}_{h}-\operatorname{Id}-\widetilde{\alpha}_{h}\right\|_{C^{1}(\mathbb{R})}<\varepsilon, \quad\left\|\widetilde{\psi}_{b}-\operatorname{Id}-\widetilde{\alpha}_{b}\right\|_{C^{1}(\mathbb{R})}<\varepsilon .
$$

Il existe donc $\delta>0$ tel que $\widetilde{\psi}_{h}$ et $\widetilde{\psi}_{b}$ s'étendent en deux applications univalentes sur $\widetilde{A}_{2 \delta}$ vérifiant

$$
\left\|\widetilde{\psi}_{h}-\operatorname{Id}-\widetilde{\alpha}_{h}\right\|_{C^{1}\left(\widetilde{A}_{2 \delta}\right)}<\varepsilon, \quad\left\|\widetilde{\psi}_{b}-\operatorname{Id}-\widetilde{\alpha}_{b}\right\|_{C^{1}\left(\widetilde{A}_{2 \delta}\right)}<\varepsilon .
$$

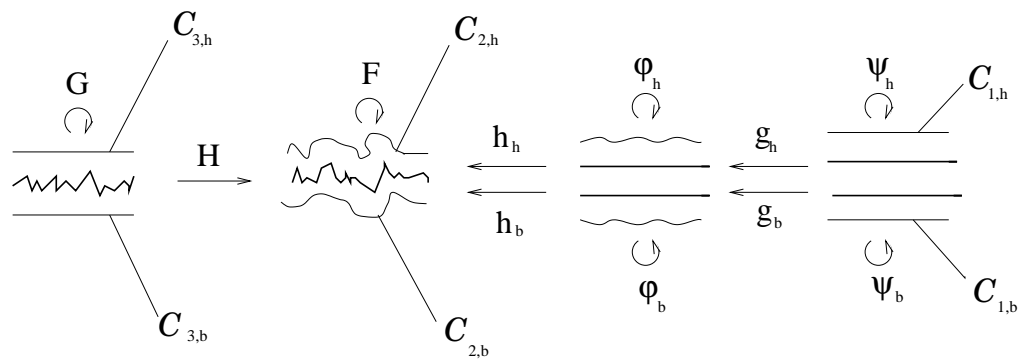

Posons $\mathcal{C}_{1, h}=\{z \in \mathbb{C} / \mathbb{Z} \mid \operatorname{Im} z=\delta\}$ et $\mathcal{C}_{1, b}=\{z \in \mathbb{C} / \mathbb{Z} \mid \operatorname{Im} z=-\delta\}$. Notons $g_{h}$ et $g_{b}$ les éléments de Diff ${ }^{\omega}\left(\mathbb{T}^{1}\right)$ dont $\widetilde{g}_{h}$ et $\widetilde{g}_{b}$ sont des relevés. Quitte à diminuer $\delta$, on peut supposer que $h_{h} \circ g_{h}$ et $h_{b} \circ g_{b}$ s'étendent en deux applications univalentes définies respectivement sur $\{z \in \mathbb{C} \mid 0<$ $\operatorname{Im} z<2 \delta\}$ et sur $\{z \in \mathbb{C} \mid-2 \delta<\operatorname{Im} z<0\}$, à valeurs dans $V$. Posons alors $\mathcal{C}_{2, h}=h_{h} \circ g_{h}\left(\mathcal{C}_{1, h}\right)$ et $\mathcal{C}_{2, b}=h_{b} \circ g_{b}\left(\mathcal{C}_{1, b}\right)$.

Considérons l'anneau $\mathcal{A}$ compris entre $\mathcal{C}_{2, b}$ et $\mathcal{C}_{2, h}$, et notons $m$ son module. Considérons une application uniformisante $H$ de $A_{m / 2}$ dans $\mathcal{A}$, et posons $G=H^{-1} \circ F \circ H$. Posons $\mathcal{C}_{3, h}=\{z \in \mathbb{C} / \mathbb{Z} \mid \operatorname{Im} z=m / 2\}$ et $\mathcal{C}_{3, b}=\{z \in \mathbb{C} / \mathbb{Z} \mid \operatorname{Im} z=-m / 2\}$. L'application $H^{-1} \circ h_{h} \circ g_{h}$ envoie tout point proche de $\mathcal{C}_{1, h}$ sur un point proche de $\mathcal{C}_{3, h}$; elle se prolonge donc par réflexion de Schwarz en une application $L_{h}$, univalente sur $\{z \in \mathbb{C} / \mathbb{Z} \mid 0<$ $\operatorname{Im} z<2 \delta\}$, et qui vérifie $L_{h}\left(\mathcal{C}_{1, h}\right)=\mathcal{C}_{3, h}$. De même, $H^{-1} \circ h_{b} \circ g_{b}$ se prolonge en une application $L_{b}$, univalente sur $\{z \in \mathbb{C} / \mathbb{Z} \mid-2 \delta<\operatorname{Im} z<0\}$, et qui vérifie $L_{b}\left(\mathcal{C}_{1, b}\right)=\mathcal{C}_{3, b}$.

Soient $\widetilde{H}, \widetilde{L}_{h}$, et $\widetilde{L}_{b}$ des relevés respectifs de $H, L_{h}$, et $L_{b}$. Posons $\widetilde{G}=$ $\widetilde{H}^{-1} \circ \widetilde{F} \circ \widetilde{H}$. L'application $z \mapsto \widetilde{G}(z)-z$ passe au quotient et définit une application holomorphe dans un voisinage de l'adhérence de $A_{m / 2}$ dans $\mathbb{C} / \mathbb{Z}$. Posons

$$
I_{h}=\int_{\mathcal{C}_{3, h}}(\widetilde{G}(z)-z) d z, \quad I_{b}=\int_{\mathcal{C}_{3, b}}(\widetilde{G}(z)-z) d z
$$

D'après la formule de Cauchy, on a $I_{h}-I_{b}=0$. Le lemme suivant mène donc clairement à une contradiction. 
LEMME 1.2.1. Il existe une constante $\varepsilon_{0}$ telle que, si $\varepsilon<\varepsilon_{0}$, alors $\operatorname{Re} I_{h}>\left[\widetilde{\alpha}_{h}\right]-1$ et $\operatorname{Re} I_{b}<\left[\widetilde{\alpha}_{b}\right]+2\left(\left[\widetilde{\alpha}_{h}\right]\right.$ et $\left[\widetilde{\alpha}_{b}\right]$ désignent les parties entières de $\widetilde{\alpha}_{h}$ et $\left.\widetilde{\alpha}_{b}\right)$.

Démonstration. L'idée : La méthode pour estimer ces intégrales consiste à "oublier" la dynamique de $F$, ainsi que celles de $\varphi_{h}$ et $\varphi_{b}$, et à travailler uniquement avec $\psi_{h}$ et $\psi_{b}$, dont les dynamiques sont reliées à celle de $G$ par $L_{h}$ et $L_{b}$. Or, l'application $L_{h}$ (par exemple) envoie la droite horizontale $\mathcal{C}_{1, h}$ sur la droite horizontale $\mathcal{C}_{3, h}$; sa dérivée est donc "presque réelle" au voisinage de $\mathcal{C}_{1, h}$, en particulier sur $\mathcal{C}_{1, h} \cup \psi_{h}\left(\mathcal{C}_{1, h}\right)$ (grâce à une estimée de Koebe), ce qui suffira pour conclure.

La démonstration : Pour montrer l'estimée sur Re $I_{h}$, il suffit d'établir que, pour tout $z \in \mathcal{C}_{3, h}$, on a $\operatorname{Re}\left(\widetilde{G}(z)-z-\left[\widetilde{\alpha}_{h}\right]+1\right)>0$. Fixons $z \in \mathcal{C}_{3, h}$ quelconque, et posons $\zeta=L_{h}^{-1}(z)\left(\zeta \in \mathcal{C}_{1, h}\right)$. Alors,

$$
\begin{aligned}
\widetilde{G}(z) & -z-\left[\widetilde{\alpha}_{h}\right]+1=\widetilde{L}_{h}\left(\widetilde{\psi}_{h}(\zeta)-\left[\widetilde{\alpha}_{h}\right]+1\right)-\widetilde{L}_{h}(\zeta) \\
& \left.=\int_{0}^{1} D \widetilde{L}_{h}\left(\zeta+t\left(\widetilde{\psi}_{h}(\zeta)-\left[\widetilde{\alpha}_{h}\right]+1-\zeta\right)\right)\left(\widetilde{\psi}_{h}(\zeta)-\left[\widetilde{\alpha}_{h}\right]+1-\zeta\right)\right) d t .
\end{aligned}
$$

Or, d'une part, $\widetilde{\psi}_{h}(\zeta)-\zeta-\widetilde{\alpha}_{h}<\varepsilon$, ce qui montre que

$$
\arg \left(\widetilde{\psi}_{h}(\zeta)-\left[\alpha_{h}\right]+1-\zeta\right)<\arcsin \varepsilon .
$$

D'autre part, soit $\zeta_{0} \in \mathbb{R} / \mathbb{Z}$ tel que $\operatorname{Re} \zeta_{0}=\operatorname{Re} \zeta$ (c'est-à-dire que $\zeta=\zeta_{0}+i \delta$ ). Alors,

$$
\left|\widetilde{\psi}_{h}(\zeta)-\widetilde{\psi}_{h}\left(\zeta_{0}\right)-i \delta\right|<\varepsilon \delta
$$

Or, puisque $D \widetilde{L}_{h \mid \mathcal{C}_{1, h}} \in \mathbb{R}$ et que $\widetilde{L}_{h}$ est univalente $\operatorname{sur}\left\{z \in \mathbb{C} / \mathbb{Z} \mid \operatorname{dist}\left(z, \mathcal{C}_{1, h}\right)\right.$ $<\delta\}$, on déduit d'une estimée de fonction univalente (Koebe) que

$$
\sup _{\operatorname{dist}\left(\xi, \mathcal{C}_{1, h}\right)<\varepsilon \delta} \arg \left(D \widetilde{L}_{h}(\xi)\right)<\varepsilon^{\prime}(\varepsilon)
$$

où $\varepsilon^{\prime}(\varepsilon) \rightarrow 0$ lorsque $\varepsilon \rightarrow 0$.

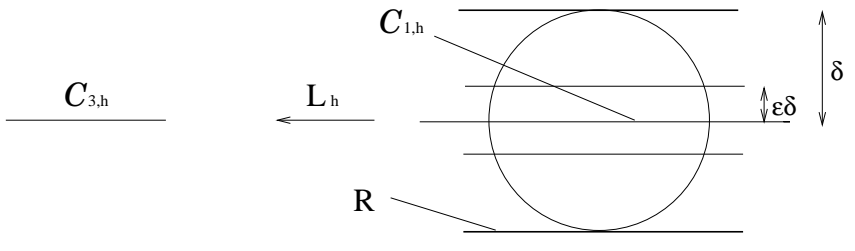

On en déduit que

$$
\arg \left(\widetilde{G}(z)-z-\left[\alpha_{h}\right]+1\right)<\arcsin \varepsilon+\varepsilon^{\prime}(\varepsilon),
$$

ce qui démontre l'estimée sur Re $I_{h}$; l'estimée sur Re $I_{b}$ s'établit de la même manière. 
Ceci démontre donc le lemme 1.2.1 (et, en même temps, la proposition 1.2.1 dans le cas où $\widetilde{\alpha}_{h}$ et $\widetilde{\alpha}_{b}$ sont tous deux irrationnels).

DEUXiÈme CAS : $\widetilde{\alpha}_{h}$ est irrationnel, $\widetilde{\alpha}_{b}$ est rationnel. Puisque $\widetilde{\alpha}_{h}$ est irrationnel, on peut effectuer, à partir de la dynamique de $F$ dans la composante connexe $U_{h}$ de $\mathbb{C} / \mathbb{Z} \backslash K$ située au-dessus de $K$, les mêmes constructions que précédemment; on construit ainsi (en gardant les mêmes notations) $g_{h}, \psi_{h}$, $\mathcal{C}_{1, h}, \mathcal{C}_{2, h}$

D'autre part, puisque $\widetilde{\alpha}_{h} \neq \widetilde{\alpha}_{b}, F_{\mid K}$ n'est pas racine de l'identité; on peut donc appliquer le lemme 1.1.2 à la paire $\left(U_{b}, F\right)\left(U_{b}\right.$ designe la composante connexe de $\overline{\mathbb{C} / \mathbb{Z}} \backslash K$ située au-dessous de $K$ ). Soit $\mathcal{C}_{b}^{\prime}$ une courbe invariante par $F$ donnée par ce lemme. On peut supposer que $\mathcal{C}_{b}^{\prime}$ sépare les deux bouts de $\mathbb{C} / \mathbb{Z}$. Notons $\mathcal{A}$ l'ensemble formé des points situés au-dessous de $\mathcal{C}_{2, h}$ et appartenant à la composante connexe de $\overline{\mathbb{C} / \mathbb{Z}} \backslash \mathcal{C}_{b}^{\prime}$ contenant $+i \infty$; puisque $\mathcal{C}_{b}^{\prime}$ est connexe, $\mathcal{A}$ est un sous-anneau de $\mathbb{C} / \mathbb{Z}$; notons $m$ son module, introduisons encore une uniformisante $H: A_{m / 2} \rightarrow \mathcal{A}$, et posons $G=H^{-1} \circ F \circ H$.

La suite de la démonstration fonctionne de la même manière que précédemment. La courbe $\mathcal{C}_{3, b}$ est cette fois-ci exactement invariante par $G$ (ce qui permet de prolonger $G$ par réflexion de Schwarz au voisinage de cette courbe) et $\mathcal{C}_{3, h}$ est toujours quasi-invariante. On établit (avec les mêmes notations) que $I_{h}>\left[\alpha_{h}\right]-1$ comme précédemment, et le fait que $I_{b}<\left[\alpha_{b}\right]+2$ est évident. On aboutit donc encore à une contradiction avec la formule de Cauchy.

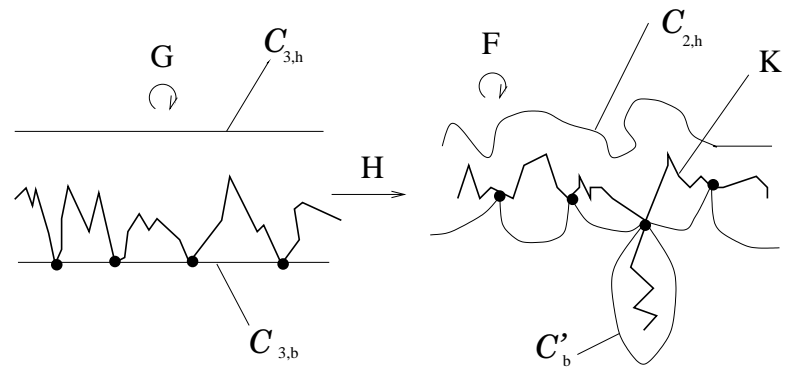

Troisième CAS : $\widetilde{\alpha}_{h}$ et $\widetilde{\alpha}_{b}$ sont tous deux rationnels. Dans ce cas, on peut appliquer le lemme 1.1.2 dans chacune des deux composantes connexes de $\mathbb{C} / \mathbb{Z} \backslash K\left(F_{\mid K}\right.$ n'est pas racine de l'identité). Considérons deux courbes $\mathcal{C}_{2, h}$ et $\mathcal{C}_{2, b}$ (données par ce lemme) invariantes par $F$, situées respectivement au-dessus et au-dessous de $K$.

Si $\mathcal{C}_{2, h} \cap \mathcal{C}_{2, b}=\emptyset$, alors on peut encore considérer l'anneau compris entre $\mathcal{C}_{2, b}$ et $\mathcal{C}_{2, h}$ et raisonner comme précédemment.

Si en revanche $\mathcal{C}_{2, h} \cap \mathcal{C}_{2, b} \neq \emptyset$, alors, d'après le lemme 1.1.2, elles se rencontrent en un point périodique de $K$ (noté $z_{0}$ ). Posons $\widetilde{\alpha}_{h}=p / q$ et 
$\widetilde{\alpha}_{b}=p^{\prime} / q^{\prime}$; alors, puisque les relevés des représentations conformes $h_{h}$ et $h_{b}$ commutent aux translations entières, on a $\widetilde{F}^{q}\left(z_{0}\right)=z_{0}+p$ et $\widetilde{F}^{q^{\prime}}\left(z_{0}\right)=$ $z_{0}+p^{\prime}$, ce qui entraîne que $p / q=p^{\prime} / q^{\prime}$.

Ceci termine la démonstration de la proposition 1.2.1.

Ceci fournit en particulier une nouvelle démonstration, peut-être plus simple, du résultat suivant :

Corollaire 1.2.1 ([PM1]). Si $\mathbb{T}^{1} \subset K$ et si $F\left(\mathbb{T}^{1}\right)=\mathbb{T}^{1}\left(\right.$ donc $\widetilde{F}_{\mid \mathbb{R}} \in$ $\left.D^{\omega}\left(\mathbb{T}^{1}\right)\right)$, alors $\widetilde{\alpha}_{h}=\widetilde{\alpha}_{b}=\varrho\left(\widetilde{F}_{\mid \mathbb{R}}\right)$.

Démonstration. Il suffit d'appliquer la proposition précédente aux compacts $K_{h}=K \cap\{z \in \mathbb{C} / \mathbb{Z} \mid \operatorname{Im} z \geq 0\}$ et $K_{b}=K \cap\{z \in \mathbb{C} / \mathbb{Z} \mid \operatorname{Im} z$ $\leq 0\}$.

La proposition 1.2.1 montre que l'on peut naturellement associer à $K$ un nombre de rotation. La même idée va nous permettre d'établir la

Proposition 1.2.2. Si le nombre de rotation associé à $K$ est irrationnel, alors $F$ n'a pas d'orbite périodique sur $K$.

(Il s'agit de l'assertion (1) du théorème 2 de l'introduction dans le cas annulaire.)

Démonstration. Supposons au contraire que $F_{\mid K}$ admette une orbite périodique. Quitte à remplacer $F$ par un de ses itérés, on peut supposer que $F$ admet un point fixe $z_{0}$ sur $K$. Soit $\widetilde{F}$ un relevé de $F$ par le revêtement $\pi: \mathbb{C} \rightarrow \mathbb{C} / \mathbb{Z}$ qui fixe les images réciproques de $z_{0}$. Supposons que le nombre de rotation associé à ce relevé et à l'ensemble invariant $\pi^{-1}(K)$ soit, par exemple, strictement positif; quitte à remplacer encore une fois $\widetilde{F}$ par un de ses itérés, on peut supposer qu'il est plus grand que 1.

Comme lors de la démonstration précédente, on construit deux courbes $\mathcal{C}_{2, h}$ et $\mathcal{C}_{2, b}$, situées de part et d'autre de $K$, quasi-invariantes par $F$. Soit $H$ une application uniformisante de l'anneau compris entre ces deux courbes, et $\widetilde{H}$ un relevé de $H$; posons $\widetilde{G}=\widetilde{H}^{-1} \circ \widetilde{F} \circ \widetilde{H}$. On montre alors (comme précédemment) que $\operatorname{Re}(\widetilde{G}(z)-z)$ est strictement positif sur $\mathcal{C}_{3, h}$ et $\mathcal{C}_{3, b}$. D'autre part, $\widetilde{G}\left(\widetilde{H}^{-1}\left(z_{0}\right)\right)=\widetilde{H}^{-1}\left(z_{0}\right)$, ce qui est en contradiction avec le principe du maximum.

1.3. Compact plein. Considérons à présent une paire $(K, F) \in \mathcal{K}$ quelconque, et supposons que $K$ soit plein. Soit $h$ une représentation conforme de $\overline{\mathbb{C}} \backslash \overline{\mathbb{D}}$ dans $\overline{\mathbb{C}} \backslash K$. L'application $h^{-1} \circ F \circ h$ se prolonge par réflexion de Schwarz en une application $\varphi$ univalente au voisinage de $\mathbb{S}^{1}$, dont la restriction à $\mathbb{S}^{1}$ définit un difféomorphisme analytique du cercle; notons $\alpha$ son nombre de rotation. La proposition suivante correspond à l'assertion (1) du théorème 2 dans le cas d'un compact plein. 
Proposition 1.3.1. Supposons que a soit irrationnel. Alors, il existe un unique point fixe $z_{0}$ sur $K$ qui vérifie $F^{\prime}\left(z_{0}\right)=e^{2 i \pi \alpha}$. De plus, $F_{\mid K}$ n'a pas de point périodique autre que $z_{0}$.

Démonstration. L'existence d'un point fixe pour $F$ sur $K$ découle du théorème du point fixe de Cartwright-Littlewood ([C,L]) : Tout homéomorphisme du plan préservant l'orientation et laissant invariant un ensemble compact connexe plein admet un point fixe sur cet ensemble. Pour pouvoir appliquer ce théorème, il suffit donc de vérifier qu'il existe un homéomorphisme $\widehat{F}$ de $\mathbb{C}$ qui coïncide avec $F$ au voisinage de $K$; il existe alors un point $z_{0} \in K$ fixe par $\widehat{F}$, donc par $F$.

Posons $F^{\prime}\left(z_{0}\right)=e^{2 i \pi \beta}, \beta \in \mathbb{C} / \mathbb{Z}\left(F^{\prime}\left(z_{0}\right) \neq 0\right.$ puisque $F$ est univalente). Le lemme 1.1.4 entraîne que $\beta$ appartient à $\mathbb{R} / \mathbb{Z}$, et même à $(\mathbb{R} \backslash \mathbb{Q}) / \mathbb{Z}$. Notons $\theta$ l'application $\mathbb{C} / \mathbb{Z} \rightarrow \mathbb{C} \backslash\left\{z_{0}\right\}, z \mapsto z_{0}+e^{2 i \pi z}$. Posons $K_{1}=$ $\theta^{-1}\left(K \backslash\left\{z_{0}\right\}\right)$ et $F_{1}=\theta^{-1} \circ F \circ \theta$ (l'application $F_{1}$ est définie et univalente dans un voisinage de $K_{1}$ dans $\left.\mathbb{C} / \mathbb{Z}\right)$.

Considérons une représentation conforme $h_{b}: \mathbb{H}^{*} / \mathbb{Z} \rightarrow \mathbb{C} / \mathbb{Z} \backslash K_{1}$. L'application $h_{b}^{-1} \circ F_{1} \circ h_{b}$ se prolonge par réflexion de Schwarz en une application $\varphi_{b}$ univalente au voisinage de $\mathbb{T}^{1}$, dont la restriction à $\mathbb{T}^{1}$ définit un difféomorphisme analytique du cercle de nombre de rotation $\alpha$.

Soit $\widetilde{F}_{1}$ un relevé de $F_{1}$ et $\widetilde{h}_{b}$ un relevé de $h_{b}$ par le revêtement $\pi: \mathbb{C} \rightarrow$ $\mathbb{C} / \mathbb{Z}$. L'application $\widetilde{\varphi}_{b}=\widetilde{h}_{b}^{-1} \circ \widetilde{F}_{1} \circ \widetilde{h}_{b}$ définit (après prolongement) un relevé de $\varphi_{b}$. Notons $\widetilde{\alpha} \in \mathbb{R}$ son nombre de rotation, qui vérifie $\pi(\widetilde{\alpha})=\alpha$. Par ailleurs, la limite $\lim _{\operatorname{Im} z \rightarrow+\infty}\left(\widetilde{F}_{1}(z)-z\right)$ est bien définie; notons-la $\widetilde{\beta}$; on a alors $\pi(\widetilde{\beta})=\beta$.

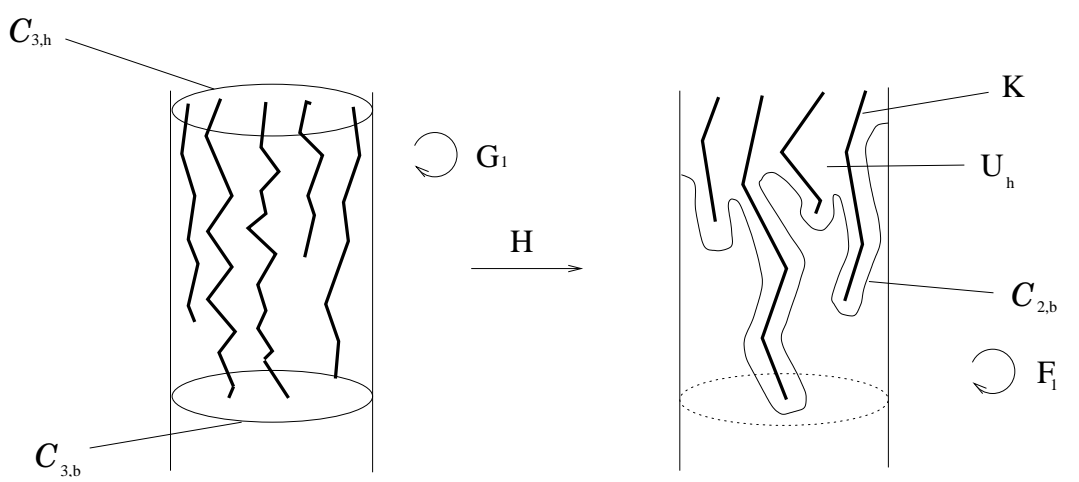

Assertion. $\widetilde{\beta}=\widetilde{\alpha}$ (ce qui entraine, en particulier, que $\alpha=\beta$ ).

La démarche pour démontrer cette assertion est très similaire à celle utilisée pour la démonstration de la proposition 1.2.1. Raisonnons par l'absurde et supposons $\widetilde{\beta} \neq \widetilde{\alpha}$; on peut alors supposer que $\widetilde{\alpha}>1$ et que $\widetilde{\beta}<$ -1 . Construisons, de la méme manière que dans la démonstration de la 
proposition 1.2.1, une courbe $\mathcal{C}_{2, b}$ quasi-invariante par $F_{1}$; notons $U_{h}$ l'ouvert situé au-dessus de $\mathcal{C}_{2, b}$ dans $\mathbb{C} / \mathbb{Z}$, et considérons une représentation conforme $H: \mathbb{H} / \mathbb{Z} \cup\{+i \infty\} \rightarrow U_{h} \cup\{+i \infty\}$; soit $\widetilde{H}$ un relevé de $H$. L'application $\widetilde{G}_{1}=\widetilde{H}^{-1} \circ \widetilde{F}_{1} \circ \widetilde{H}$ peut être prolongée en une application univalente au voisinage de $\mathbb{H} \cup \mathbb{R}$.

Soit $\Delta>0$ très grand; posons $\mathcal{C}_{3, b}=\mathbb{R} / \mathbb{Z}, \mathcal{C}_{3, h}=\{z \in \mathbb{C} / \mathbb{Z} \mid \operatorname{Im} z=\Delta\}$,

$$
I_{h}=\int_{\mathcal{C}_{3, h}}\left(\widetilde{G}_{1}(z)-z\right) d z, \quad I_{b}=\int_{\mathcal{C}_{3, b}}\left(\widetilde{G}_{1}(z)-z\right) d z .
$$

Alors on montre (toujours de la même manière) que $\operatorname{Re} I_{b}>[\widetilde{\alpha}]-1$; d'autre part, si $\Delta$ est assez grand, $I_{h}$ est arbitrairement proche de $\widetilde{\beta}$, en particulier $\operatorname{Re} I_{h}<0$. On aboutit donc encore à une contradiction avec la formule de Cauchy. L'assertion $\widetilde{\beta}=\widetilde{\alpha}$ est donc démontrée.

Enfin, la non-existence de point périodique par $F$ sur $K \backslash\left\{z_{0}\right\}$ se démontre de la même manière que dans le cas d'un compact annulaire (voir la proposition 1.2.2), en construisant deux courbes quasi-invariantes et en appliquant le principe du maximum. Ceci termine la démonstration de la proposition 1.3.1.

1.4. Compact ni plein, ni annulaire. Considérons une paire $(K, F) \in$ $\mathcal{K}$ quelconque; soit $V$ un voisinage ouvert connexe de $K$ tel que $F$ soit univalente sur $V$; faisons les hypothèses suivantes :

(a) $\mathbb{C} \backslash K$ a un nombre fini, supérieur ou égal à 3 , de composantes connexes (notons $n$ ce nombre).

(b) Pour toute composante connexe $U$ de $\mathbb{C} \backslash K$, on a $F(V \cap U) \subset U$.

Notons $U_{1}, \ldots, U_{n}$ les composantes connexes de $\overline{\mathbb{C}} \backslash K$. Pour tout $i \in\{1, \ldots$ $\ldots, n\}$, fixons une représentation conforme $\theta_{i}: \mathbb{H} / \mathbb{Z} \cup\{+i \infty\} \rightarrow U_{i}$. L'application $\theta_{i}^{-1} \circ F \circ \theta_{i}$ se prolonge par réflexion de Schwarz en une application $\varphi_{i}$ univalente au voisinage de $\mathbb{T}^{1}$, dont la restriction à $\mathbb{T}^{1}$ définit un élément de Diff ${ }^{\omega}\left(\mathbb{T}^{1}\right)$; notons $\alpha_{i} \in \mathbb{R} / \mathbb{Z}$ son nombre de rotation ( $\alpha_{i}$ ne dépend pas du choix de $\theta_{i}$ ).

L'objectif de cette section est de démontrer la proposition suivante (qui correspond à la proposition 2 de l'introduction) :

Proposition 1.4.1. Pour tout $i \in\{1, \ldots, n\}, \alpha_{i}=0$.

Débarassons-nous pour commencer du cas où $F_{\mid K}$ est racine de l'identité :

LEMmE 1.4.1. Si $F_{\mid K}^{q}=\mathrm{Id}$, alors $F_{\mid K}=\mathrm{Id}$.

Démonstration. Raisonnons par l'absurde et supposons que $F_{\mid K}^{q}=$ Id avec $q \geq 2$, et $F_{\mid K} \neq \mathrm{Id}$. Alors, pour tout $i \in\{1, \ldots, n\}$, on a $\varphi_{i}^{q}=\mathrm{Id}$, et $\alpha_{i}$ est non nul dans $\mathbb{R} / \mathbb{Z}$ (sinon on aurait $\varphi_{i}=$ Id et donc $F_{\mid K}=\mathrm{Id}$ ). Fixons $x_{1} \in U_{1}$ et $x_{2} \in U_{2}$ (quelconques) et $M$ une transformation de 
Möbius vérifiant $M(0)=x_{1}$ et $M(\infty)=x_{2}$. Considérons le revêtement $R: \mathbb{C} \rightarrow \mathbb{C} \backslash\left\{x_{1}, x_{2}\right\}, z \mapsto M\left(e^{2 i \pi z}\right)$. Remarquons que $R^{-1}\left(U_{3}\right)$ n'est pas connexe. Soit $\widetilde{U}_{3}$ une composante connexe quelconque de $R^{-1}\left(U_{3}\right)$. Soit $\widetilde{F}$ un relevé de $F$ par le revêtement $R(\widetilde{F}$ est définie sur un voisinage connexe $W$ de $\left.R^{-1}(K)\right)$, et vérifiant $\widetilde{F}\left(\widetilde{U}_{3} \cap W\right) \subset \widetilde{U}_{3}$ (puisque $F\left(U_{3} \cap V\right) \subset U_{3}$, un tel relevé existe bien).

Puisque $\alpha_{1}$ est non nul dans $\mathbb{R} / \mathbb{Z}$, il existe un entier $p$ non nul tel que $\widetilde{F}^{q}-p=$ Id sur les points de $R^{-1}\left(U_{1}\right)$ proches de $R^{-1}(K)$. Ceci impose que $\widetilde{F}^{q}-p=\mathrm{Id}$ sur tout un voisinage de $R^{-1}(K)$ dans $\mathbb{C}$. Mais d'autre part, puisque $\widetilde{F}\left(\widetilde{U}_{3} \cap W\right) \subset \widetilde{U}_{3}$, on a $\widetilde{F}^{q}=$ Id sur les points de $\widetilde{U}_{3}$ proches de $R^{-1}(K)$, d'où une contradiction.

Ceci démontre la proposition 1.4.1 dans le cas où $F_{\mid K}$ est racine de l'identité, et nous supposerons dorénavant que $F_{\mid K}$ n'est pas racine de l'identité.

La suite de la démonstration se fait en trois étapes :

LeMme 1.4.2. Un au moins des $\alpha_{i}, i \in\{1, \ldots, n\}$, est nul.

Dém o nstration. Raisonnons par l'absurde et supposons que tous les $\alpha_{i}$ soient non nuls. Soit $F_{1}$ un homéomorphisme de $\overline{\mathbb{C}}$ qui coïncide avec $F$ au voisinage de $K$. Pour tout $i \in\{1, \ldots, n\}$, soit $V_{i}$ un domaine de Jordan satisfaisant $\overline{V_{i}} \subset U_{i}$, et suffisamment grand pour contenir tous les points fixes de $F_{1}$ inclus dans $U_{i}$. Puisque $\alpha_{i} \neq 0$, l'indice de Lefschetz $i\left(F_{1}, V_{i}\right)$ est égal à 1 . Notons $\mathcal{F}$ l'ensemble des points de $K$ qui sont fixés par $F$; puisque $F_{\mid K}$ n'est pas l'identité, cet ensemble est fini; en outre, pour tout $z \in \mathcal{F}$, puisque $F$ est univalente au voisinage de $z$, l'indice de Lefschetz $i\left(F_{1}, z\right)$ est égal à 1. D'après la formule de Lefschetz, on a

$$
2=\sum_{z \in \mathcal{F}} i\left(F_{1}, z\right)+\sum_{i=1}^{n} i\left(F_{1}, V_{i}\right)=\operatorname{Card} \mathcal{F}+n \geq 3,
$$

d'où une contradiction.

Poursuivons la démonstration de la proposition 1.4.1. Notons $p$ le nombre de composantes connexes $U_{i}$ de $\overline{\mathbb{C}} \backslash K$ pour lesquelles $\alpha_{i}$ est rationnel. D'après le lemme précédent, on a $1 \leq p \leq n$. Supposons, sans perte de généralité, que $\alpha_{1}, \ldots, \alpha_{p} \in \mathbb{Q} / \mathbb{Z}$ et $\alpha_{p+1}, \ldots, \alpha_{n} \in(\mathbb{R} \backslash \mathbb{Q}) / \mathbb{Z}$.

Fixons $i \in\{1, \ldots, p\}$ quelconque. Puisque $\alpha_{i} \in \mathbb{Q} / \mathbb{Z}$ et que $F_{\mid K}$ n'est pas racine de l'identité, on peut appliquer le lemme 1.1.2 à la dynamique de $F$ dans $U_{i}$. Rappelons que $\theta_{i}: \mathbb{H} / \mathbb{Z} \cup\{+i \infty\} \rightarrow U_{i}$ désigne une représentation conforme de $U_{i}$ et que $\varphi_{i}$ désigne l'application $\theta_{i}^{-1} \circ F \circ \theta_{i}$, prolongée par réflexion de Schwarz.

Notons $\mathcal{C}_{i}=\theta_{i}\left(\mathcal{C}_{i}^{\prime}\right)$ la courbe $F$-invariante donnée par le lemme 1.1.2 appliqué à la paire $\left(U_{i}, F\right)$ (on a prolongé $\theta_{i}$ aux points de $\mathcal{C}_{i}^{\prime}$ qui appartiennent 
à $\mathbb{R} / \mathbb{Z}$ par ses limites non tangentielles en ces points). Notons $W_{i}$ la réunion des composantes connexes de $\mathbb{C} / \mathbb{Z} \backslash\left(\mathbb{R} / \mathbb{Z} \cup \mathcal{C}_{i}^{\prime}\right)$ bornées en partie imaginaire et $\Omega_{i}$ la composante connexe de $\mathbb{H} / \mathbb{Z} \backslash \mathcal{C}_{i}^{\prime}$ non bornée en partie imaginaire; clairement, $\varphi_{i}\left(W_{i}\right)=W_{i}$; notons $X_{i}=\theta_{i}\left(W_{i}\right)$ et $U_{i}^{\prime}=\theta_{i}\left(\Omega_{i}\right)$; alors, $F\left(X_{i}\right)=X_{i}$ (on peut supposer que $X_{i}$ est inclus dans $V$ ) et $F\left(U_{i}^{\prime} \cap V\right) \subset U_{i}^{\prime}$. Posons $\mathcal{C}=\bigcup_{i=1}^{p} \mathcal{C}_{i}, X=\bigcup_{i=1}^{p} X_{i}$, et $\widehat{K}=K \cup \mathcal{C} \cup X$. L'ensemble $\widehat{K}$ est encore un ensemble compact, connexe, inclus dans $V$, et vérifiant $F(\widehat{K})=\widehat{K}$; on a $\overline{\mathbb{C}} \backslash \widehat{K}=U_{1}^{\prime} \cup \ldots \cup U_{p}^{\prime} \cup U_{p+1} \cup \ldots \cup U_{n}$.

On a alors le

Lemme 1.4.3. Les $\alpha_{i}, i \in\{1, \ldots, n\}$, sont tous rationnels.

Démonstration. Raisonnons par l'absurde et supposons que les $\alpha_{i}$ ne soient pas tous rationnels, c'est-à-dire que $p<n$. Soit $\mathcal{C}_{0}$ une composante connexe de $\mathcal{C}$ quelconque, et soit $Y$ la composante connexe de $\overline{\mathbb{C}} \backslash \mathcal{C}_{0}$ qui contient $U_{p+1}$.

Montrons que $F(Y \cap V) \subset Y$. Puisque $Y$ est connexe, que $V$ est connexe, et que $\partial Y \cap \partial V$ est vide, l'ensemble $Y \cap V$ est également connexe (pour s'en convaincre, on peut par exemple considérer deux points quelconques $x$ et $x^{\prime}$ de $Y \cap V$, deux chemins $\gamma_{1}$ et $\gamma_{2}$ joignant $x$ à $x^{\prime}$, respectivement dans $Y$ et dans $V$, et une équivalence d'homotopie entre $\gamma_{1}$ et $\left.\gamma_{2}\right)$. Donc $F(Y \cap V)$ est connexe, et puisque $F\left(\mathcal{C}_{0}\right)=\mathcal{C}_{0}, F(Y \cap V)$ est tout entier inclus dans une des composantes connexes de $\overline{\mathbb{C}} \backslash \mathcal{C}_{0}$; enfin, puisque $U_{p+1} \subset Y$, et que $F\left(U_{p+1} \cap V\right) \subset U_{p+1}, F(Y \cap V)$ est inclus dans $Y$.

Soit $h: \mathbb{D} \rightarrow Y$ une représentation conforme de $Y$; posons $L=h^{-1}(\widehat{K} \cap$ $Y) \cup \mathbb{S}^{1} \cup \sigma\left(h^{-1}(\widehat{K} \cap Y)\right.$ ) (où $\sigma$ désigne l'involution $\overline{\mathbb{C}} \rightarrow \overline{\mathbb{C}}, z \mapsto 1 / \bar{z}$ ), et notons $\varphi$ l'application obtenue en prolongeant $h^{-1} \circ F \circ h$ par réflexion de Schwarz. L'application $\varphi$ est univalente au voisinage de $L$, vérifie $\varphi(L)=L$, $\varphi\left(\mathbb{S}^{1}\right)=\mathbb{S}^{1}$, et $\varphi_{\mid \mathbb{S}^{1}}$ définit un élément de Diff ${ }^{\omega}\left(\mathbb{S}^{1}\right)$, de nombre de rotation rationnel. L'application $h$ se prolonge continûment en une application $\bar{h}$ : $\overline{\mathbb{D}} \rightarrow \bar{Y}$.

Soit $x$ un point de $\mathbb{S}^{1}$ périodique par $F$; notons $q$ sa période. Remarquons que le point $x$ ne peut pas appartenir à l'intérieur de $L$. En effet, raisonnons par l'absurde et supposons que $x$ appartienne à l'intérieur de $L$. Soit $M$ la composante connexe $\operatorname{de} \operatorname{int}(L)$ contenant $x$; alors, puisque $\varphi^{q}(x)=x$, on a $\varphi^{q}(M)=M$. Considérons un revêtement $\pi: \mathbb{D} \rightarrow M$, un point $x_{0} \in$ $\pi^{-1}(\{x\})$ quelconque, et un relevé $\psi$ de $\varphi_{\mid M}^{q}$ au disque $\mathbb{D}$ par le revêtement $\pi$, vérifiant $\psi\left(x_{0}\right)=x_{0}$. Alors $\psi$ est une transformation de Möbius, et puisque $\psi^{\prime}\left(x_{0}\right)=\varphi^{\prime}\left(x_{0}\right) \in \mathbb{R}_{+}^{*}$, on a $\psi^{\prime}\left(x_{0}\right)=1$, donc $\psi=\mathrm{Id}, \varphi_{\mid M}^{q}=\mathrm{Id}$, et finalement $F^{q}=\mathrm{Id}$, ce qui est contraire aux hypothèses.

Il existe donc une composante connexe $Z$ de $\overline{\mathbb{C}} \backslash L$, incluse dans $\mathbb{D}$, telle que $x \in \partial Z$. Clairement, $\varphi\left(Z \cap h^{-1}(V)\right) \subset Z$ et, d'après le lemme 1.1.4, le 
nombre de rotation associé à la dynamique de $\varphi$ dans $Z$ ne peut pas être irrationnel; il existe donc $j \in\{1, \ldots, p\}$ tel que $Z=h^{-1}\left(U_{j}^{\prime}\right)$. On a $\partial Z \subset \overline{\mathbb{D}}$; mais d'autre part, $\partial U_{j}^{\prime} \subset \mathcal{C}$, et $\partial U_{j}^{\prime} \cap \mathcal{C}_{0}$ (qui contient $\bar{h}(x)$ ) est non vide; donc $\partial U_{j}^{\prime} \subset \mathcal{C}_{0}$. On en déduit que $\partial Z=\partial \mathbb{D}$. Finalement, $Z=\mathbb{D}$, d'où une contradiction. Ceci démontre le lemme 1.4.3.

Conservons les notations introduites avant l'énoncé du lemme 1.4.3 (en particulier l'ensemble $\widehat{K}$ ); nous savons maintenant que $p=n$ et que $\overline{\mathbb{C}} \backslash \widehat{K}=$ $U_{1}^{\prime} \cup \ldots \cup U_{n}^{\prime}$; en particulier, $\partial \widehat{K}=\mathcal{C}_{1} \cup \ldots \cup \mathcal{C}_{n}$.

Le lemme suivant conclut la démonstration de la proposition 1.4.1.

Lemme 1.4.4. Les $\alpha_{i}, i \in\{1, \ldots, n\}$, sont tous nuls.

Démonstration. Raisonnons encore une fois par l'absurde et supposons que l'un des $\alpha_{i}$, par exemple $\alpha_{1}$, soit non nul (dans $\mathbb{Q} / \mathbb{Z}$ ). Soit $W$ la composante connexe de $\overline{\mathbb{C}} \backslash \mathcal{C}_{1}$ qui contient $U_{2}^{\prime}$; clairement, $F(W \cap V) \subset W$; posons $\mathcal{C}_{1}^{\prime \prime}=\partial W$. Alors $\mathcal{C}_{1}^{\prime \prime} \subset \mathcal{C}_{1}$ et $F\left(\mathcal{C}_{1}^{\prime \prime}\right)=\mathcal{C}_{1}^{\prime \prime}$. Puisque $\mathcal{C}_{1}^{\prime \prime} \subset \partial W \cap \partial U_{1}^{\prime}$, le complémentaire de $\mathcal{C}_{1}^{\prime \prime}$ dans $\mathbb{C}$ n'a que deux composantes connexes $\left(\mathcal{C}_{1}^{\prime \prime}\right.$ est une réunion finie d'arcs analytiques). Puisque $n \geq 3$, une de ces deux composantes connexes (notée $X$ ) contient (au moins) deux des ouverts $U_{1}^{\prime}, \ldots, U_{n}^{\prime}$. Soit $g: \mathbb{D} \rightarrow X$ une représentation conforme de $X$. Notons $\psi$ l'application obtenue en prolongeant $g^{-1} \circ F \circ g$ par réflexion de Schwarz. La restriction de $\psi$ à $\mathbb{S}^{1}$ définit un élément de Diff ${ }^{\omega}\left(\mathbb{S}^{1}\right)$, de nombre de rotation rationnel et non nul (en effet, les arcs analytiques qui constituent $\mathcal{C}_{1}^{\prime \prime}$ ne sont pas invariants par $F$, puisque $\alpha_{1}$ est non nul).

Notons $\mathcal{U}=g^{-1}\left(X \cap\left(U_{1}^{\prime} \cup \ldots \cup U_{n}^{\prime}\right)\right)$; d'après ce qui précède, $\mathcal{U}$ a au moins deux composantes connexes. Soit $x$ un point de $\mathbb{S}^{1}$ périodique par $\psi$; notons $q$ sa période. Puisque $F_{\mid K}$ n'est pas racine de l'identité, $\psi$ n'est pas linéarisable au voisinage de $x$, donc $x \in \overline{\mathcal{U}}$. Soit $Y$ une composante connexe de $\mathcal{U}$ qui contienne $x$ dans son adhérence, et $Z$ une composante connexe de $\mathcal{U}$ quelconque, différente de $Y$. Fixons $z_{0} \in Z$ quelconque. Soit $M$ une transformation de Möbius vérifiant $M(\mathbb{D})=\mathbb{D}$ et $M(0)=z_{0}$, et soit $R$ le revêtement $\mathbb{C} \rightarrow \mathbb{C} \backslash\left\{z_{0}\right\}, z \mapsto M\left(e^{2 i \pi z}\right)$. Clairement, $R^{-1}(Y)$ n'est pas connexe, et ses composantes connexes sont bornées. Soit $\widetilde{\psi}$ un relèvement de $\psi$ à $\mathbb{C}$ qui laisse invariantes les composantes connexes de $R^{-1}(Y)$. Alors, puisque $x \in \partial Y$, l'orbite de tout point de $R^{-1}(\{x\})$ par $\widetilde{\psi}$ est bornée, ce qui est contradictoire avec le fait que le nombre de rotation de $\widetilde{\psi}_{\mathbb{R}}$ est non nul.

Ceci termine la démonstration du lemme 1.4.4, et, en même temps, de la proposition 1.4.1.

1.5. Description de la dynamique au voisinage d'un compact plein ou annulaire de nombre de rotation irrationnel. Dans cette section, nous indiquons rapidement comment certains résultats de [PM3] s'adaptent au cadre des compacts pleins ou annulaires de nombre de rotation irrationnel (c'est le 
théorème 2); nous reprenons la même démarche que dans [PM3]; le cas des compacts pleins est strictement identique, et, seul point nouveau, dans le cas des compacts annulaires, nous nous appuyons sur la proposition 1.

L'outil crucial est la proposition 1.5.1 ci-dessous.

Notons $\varrho_{\mathrm{H}}$ la métrique hyperbolique de $\mathbb{H} / \mathbb{Z} \cup\{+i \infty\}$ et posons $\widetilde{\varrho}_{\mathrm{H}}=$ $\pi_{*}\left(\varrho_{\mathrm{H}}\right)(\pi$ designe la projection canonique $\mathbb{C} \rightarrow \mathbb{C} / \mathbb{Z})$. Notons $d_{\mathrm{H}}$ la distance sur $\mathbb{H}$ induite par $\widetilde{\varrho}_{\mathrm{H}}$.

Soit $\varphi \in \operatorname{Diff}^{\omega}\left(\mathbb{T}^{1}\right)$ et $\widetilde{\varphi} \in D^{\omega}\left(\mathbb{T}^{1}\right)$ un relevé de $\varphi$; l'application $\varphi$ (resp. $\widetilde{\varphi}$ ) se prolonge en une application univalente dans un voisinage de $\mathbb{R} / \mathbb{Z}$ (resp. de $\mathbb{R}$ ) que l'on note encore $\varphi$ (resp. $\widetilde{\varphi}$ ).

Supposons que le nombre de rotation de $\widetilde{\varphi}$, noté $\widetilde{\alpha}$, soit irrationnel, et notons $\left(p_{n} / q_{n}\right)_{n \in \mathbb{N}}$ la suite de ses réduites. On a alors la

Proposition 1.5.1 ([PM3], proposition II.3). Il existe une suite $\left(\mathcal{C}_{n}\right)_{n \in \mathbb{N}}$ de courbes de Jordan incluses dans $\mathbb{H} / \mathbb{Z}$ telles que:

(i) Pour tout $n \in \mathbb{N}, \mathcal{C}_{n}$ est non homotope à un point dans $\mathbb{H} / \mathbb{Z}$ et

$$
\sup _{z \in \mathcal{C}_{n}} \operatorname{Im} z \rightarrow 0 \quad \text { lorsque } n \rightarrow+\infty \text {. }
$$

(ii) Pour tout $n \in \mathbb{N}, \varphi^{q_{n}}$ est définie sur $\mathcal{C}_{n}$ et si on pose $\widetilde{\mathcal{C}_{n}}=\pi^{-1}\left(\mathcal{C}_{n}\right)$, alors

$$
\sup _{z \in \widetilde{\mathcal{C}}_{n}} \widetilde{d}_{\mathrm{H}}\left(\widetilde{\varphi}^{q_{n}}(z), z+p_{n}\right) \rightarrow 0 \quad \text { lorsque } n \rightarrow+\infty .
$$

Toute la force de cet énoncé réside dans le fait que l'estimée sur $\widetilde{\varphi}^{q_{n}}$ fait intervenir la distance hyperbolique de $\mathbb{H} / \mathbb{Z}$, et non la distance usuelle (en quelque sorte, elle est exprimée sous une forme invariante conforme).

Nous allons voir que cette proposition entraîne sans difficulté le théorème 2 énoncé en introduction.

Considérons une paire $(K, F) \in \mathcal{K}$ quelconque. Supposons que $K$ soit plein ou annulaire et que son nombre de rotation soit irrationnel.

Idée de la démonstration du théorème 2. L'assertion (1) a déjà été établie précédemment (voir propositions 1.2.2 et 1.3.1); démontrons les assertions suivantes.

Supposons par exemple que $K$ soit annulaire (lorsque $K$ est plein, la démonstration est essentiellement la même). Pour simplifier la présentation, supposons que $K$ soit inclus dans $\mathbb{C} / \mathbb{Z}$, et sépare les deux bouts de $\mathbb{C} / \mathbb{Z}$.

Notons $U_{h}$ et $U_{b}$ les composantes connexes de $\overline{\mathbb{C} / \mathbb{Z}} \backslash K$ contenant respectivement $+i \infty$ et $-i \infty$. Notons $\widetilde{K}=\pi^{-1}(K), \widetilde{U}_{h}=\pi^{-1}\left(U_{h}\right), \widetilde{U}_{b}=\pi^{-1}\left(U_{b}\right)$ et considérons un relevé $\widetilde{F}$ de $F$ par $\pi$. Notons $\alpha$ le nombre de rotation associé à $K(\alpha \in \mathbb{R} / \mathbb{Z})$. A la dynamique de $\widetilde{F}$ sur $\widetilde{K}$ est associé un nombre de rotation $($ dans $\mathbb{R})$ noté $\widetilde{\alpha}$, vérifiant $\pi(\widetilde{\alpha})=\alpha$. Notons $\left(p_{n} / q_{n}\right)_{n \in \mathbb{N}}$ la suite des réduites de $\widetilde{\alpha}$. Notons $\varrho_{\mathrm{h}}$ la métrique hyperbolique de $U_{h} ;$ notons 
$\widetilde{\varrho}_{\mathrm{h}}=\pi_{*}\left(\varrho_{\mathrm{h}}\right), d_{\mathrm{h}}$ la distance induite par $\varrho_{\mathrm{h}}$ dans $U_{h}$, et $\widetilde{d}_{\mathrm{h}}$ la distance induite par $\widetilde{\varrho}_{h}$ dans $\widetilde{U}_{h}$.

On déduit de la proposition précédente qu'il existe une suite $\left(\mathcal{C}_{h, n}\right)_{n \in \mathbb{N}}$ de courbes de Jordan incluses dans $U_{h}$ telles que :

(i) Pour tout $n \in \mathbb{N}, \mathcal{C}_{h, n}$ est non homotope à un point dans $U_{h} \backslash\{+i \infty\}$ et $\sup _{z \in \mathcal{C}_{h, n}} d(z, K) \rightarrow 0$ lorsque $n \rightarrow+\infty$ (d désigne la distance usuelle de $\mathbb{C} / \mathbb{Z})$.

(ii) Pour tout $n \in \mathbb{N}, F^{q_{n}}$ est définie sur $\mathcal{C}_{h, n}$ et si on pose $\widetilde{\mathcal{C}}_{h, n}=$ $\pi^{-1}\left(\mathcal{C}_{h, n}\right)$, alors

$$
\sup _{z \in \widetilde{\mathcal{C}}_{h, n}} \widetilde{d}_{\mathrm{h}}\left(\widetilde{F}^{q_{n}}(z), z+p_{n}\right) \rightarrow 0 \quad \text { lorsque } n \rightarrow+\infty .
$$

En particulier,

$$
\sup _{z \in \widetilde{\mathcal{C}}_{h, n}}\left|\widetilde{F}^{q_{n}}(z)-z-p_{n}\right| \rightarrow 0 \quad \text { lorsque } n \rightarrow+\infty .
$$

Bien sûr, il existe de même une suite $\left(\mathcal{C}_{b, n}\right)_{n \in \mathbb{N}}$ de courbes de Jordan incluses dans $U_{b}$ avec les mêmes propriétés. On déduit donc du principe du maximum que

$$
\sup _{z \in \widetilde{K}}\left|\widetilde{F}^{q_{n}}(z)-z-p_{n}\right| \rightarrow 0 \quad \text { lorsque } n \rightarrow+\infty .
$$

Cette estimée montre que les itérés de $F_{\mid K}$ accumulent l'identité (c'est l'assertion (2) du théorème 2) et que

$$
\sup _{z \in \widetilde{K}}\left|\left(\widetilde{F}^{N}(z)-z\right) / N-\widetilde{\alpha}\right| \rightarrow 0 \quad \text { lorsque } N \rightarrow+\infty
$$

(il suffit d'effectuer des divisions euclidiennes successives de l'entier $N$ par les entiers $q_{n}$, qui sont les dénominateurs des réduites de $\alpha$ ). L'assertion (3) du théorème 2 , relative au fait que les points de $K$ "tournent à la vitesse $\alpha$ ", est donc établie.

Pour finir, supposons que int $K$ soit non vide, et considérons une composante connexe $U$ de int $K$; alors, puisque tout point de $K$ est récurrent, $U$ est invariant par un certain itéré $F^{j}$ de $F$. L'ouvert $U$ ne peut pas être simplement connexe (sinon $F_{\mid U}^{j}$ serait conjuguée à une transformation de Möbius du disque $\mathbb{D}$, nécessairement elliptique (puisque tous les points de $U$ sont récurrents), et $F^{j}$ aurait un point fixe dans $U$, ce qui est impossible); il s'agit donc d'un anneau qui sépare les deux bouts de $\mathbb{C} / \mathbb{Z}$, et $F_{\mid U}^{j}$ est conjuguée à la rotation d'angle $\alpha$.

Ceci termine la démonstration du théorème 2 dans le cas où $K$ est annulaire (lorsque $K$ est plein, la démonstration est essentiellement identique). 


\section{Dynamiques de type Morse-Smale}

2.1. Décomposition en pétales et démonstration du théorème 1. L'objectif de cette section est de démontrer le théorème 3 , et d'en déduire le théorème 1.

Considérons une paire $(K, F) \in \mathcal{K}$ quelconque, et notons $V$ un voisinage ouvert connexe de $K$ dans $\mathbb{C}$ tel que $F$ soit définie et univalente sur $V$. Supposons que $F_{\mid K}$ ne soit pas racine de l'identité et que le nombre de composantes connexes de $\overline{\mathbb{C}} \backslash K$ soit fini. Notons $n$ ce nombre, et $U_{1}, \ldots, U_{n}$ ces composantes. Supposons également que, pour tout $i \in\{1, \ldots, n\}$, on ait $F\left(U_{i} \cap V\right) \subset U_{i}$. Enfin, si $n=1$ ou 2 (c'est-à-dire si $K$ est plein ou annulaire), supposons que le nombre de rotation naturellement associé à $K$ est rationnel, et notons-le $p / q, p \wedge q=1, q \geq 1$; si dans le cas contraire $n \geq 3$, posons $p=0$ et $q=1$.

Nous sommes alors sous les hypothèses du théorème 3 .

Démonstration du théorème 3. Fixons $i \in\{1, \ldots, n\}$ quelconque. A la dynamique de $F$ dans $U_{i}$ au voisinage de $K$ peut être naturellement associé un difféomorphisme analytique du cercle dont le nombre de rotation vaut $p / q$ (voir la partie 1 ). On peut donc appliquer le lemme 1.1.2. On en déduit qu'il existe $k_{i} \in \mathbb{N}^{*},\left(x_{j}^{(i)}\right)_{j \in \mathbb{Z} / k_{i} q \mathbb{Z}}$ un $k_{i} q$-uplet de points de $\partial U_{i}$, et $\left(\gamma_{j}^{(i)}\right)_{j \in \mathbb{Z} / k_{i} q \mathbb{Z}}$ un $k_{i} q$-uplet de chemins analytiques $\mathbb{R} \rightarrow U_{i}$, qui satisfont à toutes les propriétés énoncées dans ce lemme. En particulier, pour tout $j \in \mathbb{Z} / k_{i} q \mathbb{Z}, x_{j}^{(i)}$ est accessible par l'extérieur de $K$ et vérifie $F^{q}\left(x_{j}^{(i)}\right)=x_{j}^{(i)}$ et $\left|\left(F^{q}\right)^{\prime}\left(x_{j}^{(i)}\right)\right| \neq 1$ ou $\left(F^{q}\right)^{\prime}\left(x_{j}^{(i)}\right)=1$. Posons

$$
\mathcal{C}_{i}=\bigcup_{j \in \mathbb{Z} / k_{i} q \mathbb{Z}}\left\{x_{j}^{(i)}\right\} \cup\left\{\gamma_{j}^{(i)}(\mathbb{R})\right\}
$$

et notons $\Omega_{i}$ la composante connexe de $\overline{\mathbb{C}} \backslash \mathcal{C}_{i}$ entièrement incluse dans $U_{i}$. Posons

$$
\mathcal{C}=\bigcup_{i=1}^{n} \mathcal{C}_{i}, \quad \widehat{K}=\overline{\mathbb{C}} \backslash\left(\bigcup_{i=1}^{n} \Omega_{i}\right) .
$$

Il est clair que $F(\widehat{K})=\widehat{K}$ et $\partial \widehat{K}=\mathcal{C}$. Remarquons que, pour chacun des lacets $\gamma_{j}^{(i)}$, l'arc $\gamma_{j}^{(i)}(\mathbb{R})$ est inclus dans le bord d'exactement deux composantes connexes de $\overline{\mathbb{C}} \backslash \mathcal{C}$, dont l'une est $\Omega_{i}$, et l'autre est une composante connexe de int $\widehat{K}$. On en déduit que le nombre de composantes connexes de int $\widehat{K}$ est fini. D'autre part, considérons une composante connexe $W$ de int $\widehat{K}$; il existe un lacet $\gamma_{j}^{(i)}$ tel que $\gamma_{j}^{(i)}(\mathbb{R})$ soit inclus dans $\partial W$; alors, puisque $F^{q}\left(\gamma_{j}^{(i)}(\mathbb{R})\right)=\gamma_{j}^{(i)}(\mathbb{R})$, on a $\gamma_{j}^{(i)}(\mathbb{R}) \subset \partial F^{q}(W)$; d'après la remarque ci-dessus, ceci entraîne que $F^{q}(W)=W$. 
Il est clair qu'il existe des points de $W$ qui convergent positivement et négativement vers $\partial W$. On en déduit, d'après le lemme 1.1.1, qu'il existe deux points $x_{\alpha}$ et $x_{\omega}$ appartenant à $\partial W$, fixes par $F^{q}$, tels que tout point de $W$ converge positivement vers $x_{\omega}$ et négativement vers $x_{\alpha}$ sous l'action de $F^{q}$. On en déduit que $\partial W$ est connexe, que $W$ est simplement connexe, et que $W$ est un pétale au sens de la définition 2 (voir l'introduction).

Finalement, int $\widehat{K}$ est la réunion d'un nombre fini de pétales invariants par $F^{q}$, et la vérification des autres conclusions du théorème 3 ne pose pas de difficulté.

Remarque sur les pétales. Soit $P$ un pétale invariant par $F$ et $h: \mathbb{D} \rightarrow P$ une représentation conforme de $P$. L'application $M=h^{-1} \circ F \circ h$ est une transformation de Möbius (non elliptique) du disque $\mathbb{D}$. Notons $y_{\alpha}$ et $y_{\omega}$ les points $\alpha$-limite et $\omega$-limite de l'orbite de tout point de $\mathbb{D}$ sous l'action de $M$.

DÉfinition 2.1.1. On dira que $P$ est régulier si et seulement si l'application $h$ se prolonge en une application $\bar{h}$ qui est :

(1) continue sur $\overline{\mathbb{D}}$,

(2) univalente sur un voisinage ouvert de $\overline{\mathbb{D}} \backslash\left\{y_{\alpha}, y_{\omega}\right\}$ dans $\mathbb{C}$.

Le lecteur pourra vérifier que les pétales construits au cours de la démonstration précédente sont réguliers (leur bord est l'adhérence de l'image de un ou deux lacets analytiques $F^{q}$-invariants, pas plus!). On peut donc renforcer légèrement l'énoncé du théorème 2 en remplaçant "pétale" par "pétale régulier".

Tout pétale régulier est de l'un des trois types dessinés en introduction, ce qui rend particulièrement explicite la topologie des compacts connexes invariants de nombre de rotation rationnel!

Démonstration du théorème 1. Considérons une paire $(K, F)$ appartenant à $\mathcal{K}$ quelconque, et supposons que :

- $K$ n'est pas plein ni annulaire de nombre de rotation irrationnel.

- $K$ n'est pas racine de l'identité.

Il nous faut montrer que la dynamique de $F$ sur $K$ est de type MorseSmale. Si le nombre de composantes connexes de $\mathbb{C} \backslash K$ est fini, ceci est une conséquence du théorème 3.

Supposons donc que le nombre de composantes connexes de $\mathbb{C} \backslash K$ soit infini. Dans ce cas, il existe, d'après la proposition 3 , une paire $(\widehat{K}, \widehat{F})$ appartenant à $\mathcal{K}$ et un homéomorphisme quasi-conforme $\phi: \mathbb{C} \rightarrow \mathbb{C}$ tels que :

(1) $\phi(K) \subset \widehat{K}, \widehat{F}(\phi(K))=\phi(K)$ et $\widehat{F} \circ \phi(z)=\phi \circ F(z)$ pour tout $z \in K$.

(2) $\mathbb{C} \backslash \widehat{K}$ a un nombre fini de composantes connexes. 
Alors, $\widehat{K}$ ne peut pas être un compact plein ou annulaire de nombre de rotation irrationnel; en effet, si c'était le cas, int $\widehat{K}$ serait un disque topologique ou un anneau topologique sur lequel la dynamique de $\widehat{F}$ serait conjuguée à celle d'une rotation irrationnelle (théorème 2), ce qui serait clairement en contradiction avec le fait que $\widehat{K}$ contient un compact connexe $\phi(K)$ vérifiant $\widehat{F}(\phi(K))=\phi(K)$ et tel que $\widehat{K} \backslash \phi(K)$ ait une infinité de composantes connexes. Puisque $\overline{\mathbb{C}} \backslash \widehat{K}$ a un nombre fini de composantes connexes, on en déduit que la dynamique de $\widehat{F}$ sur $\widehat{K}$ est de type Morse-Smale. Donc, la dynamique de $F$ au voisinage de $K$ est également de type Morse-Smale.

Ceci termine la démonstration du théorème 1 .

2.2. Compact plein ou annulaire de nombre de rotation rationnel. L'objectif de ce paragraphe est démontrer la proposition 4 .

(a) Compact plein. Considérons une paire $(K, F) \in \mathcal{K}$ telle que $K$ soit plein et telle que le nombre de rotation associé à $(K, F)$ soit rationnel. D'après le théorème de Cartwright-Littlewood (voir $\S 1.3), F$ admet un point fixe sur $K$. Supposons sans perte de généralité que 0 soit ce point fixe.

Soit $h: \overline{\mathbb{C}} \backslash \overline{\mathbb{D}} \rightarrow \overline{\mathbb{C}} \backslash K$ une représentation conforme et $\varphi$ l'élément de $\operatorname{Diff}^{\omega}\left(\mathbb{S}^{1}\right)$ obtenu en prolongeant $h^{-1} \circ F \circ h$ par réflexion de Schwarz. Notons $E$ le revêtement $\mathbb{C} \rightarrow \mathbb{C}^{*}, z \mapsto e^{2 i \pi z}$. Considérons un relevé $\widetilde{F}$ (resp. $\widetilde{h}$ ) de $F$ (resp. $h$ ) par ce revêtement, et notons $\widetilde{\varphi}$ le relevé de $\varphi$ obtenu en prolongeant $\widetilde{h}^{-1} \circ \widetilde{F} \circ \widetilde{h}$ par réflexion de Schwarz. Notons $p / q, p \wedge q=1, q \geq 1$, le nombre de rotation de $\widetilde{\varphi}$; posons $\widetilde{K}=E^{-1}(K \backslash\{0\})$.

D'après le lemme 1.1.2, $h$ admet une limite non tangentielle en tout point de $\mathbb{S}^{1}$ périodique par $\varphi$, et ces limites non tangentielles sont les seuls points périodiques de $K$ (voir $\S 2.1$ ). On en déduit que si $x$ est un point périodique de $K$ différent de 0 , alors, pour tout $\widetilde{x}$ tel que $E(\widetilde{x})=x$, on a $\widetilde{F}^{q}(\widetilde{x})=\widetilde{x}+p$; en particulier, $x$ tourne à la vitesse $p / q$ autour de 0 et $q$ est la période minimale de $x$.

D'autre part, il existe $q$ arcs qui aboutissent en 0 , qui sont soit positivement, soit négativement invariants par $F$, et qui sont permutés par $F$ avec le nombre de rotation $p / q$. On en déduit que si $\left|F^{\prime q}(0)\right|=1$, alors $F^{\prime q}(0)=1$ et, plus précisement, $F^{\prime}(0)=e^{2 i \pi p / q}$. Dans ce dernier cas, pour tout $\widetilde{z} \in \widetilde{K}$, on a

$$
\lim _{|k| \rightarrow+\infty} \operatorname{Re}\left(\widetilde{F}^{k}(\widetilde{z})-\widetilde{z}\right) / k=p / q
$$

en effet, par exemple lorsque $k \rightarrow+\infty, F^{k}(E(\widetilde{z}))$ converge soit vers 0 , soit vers un point périodique de $K$ différent de 0 , et dans les deux cas l'assertion est claire.

(b) Compact annulaire. Supposons maintenant que $K$ soit annulaire, et (toujours) de nombre de rotation $p / q$. Notons $U_{i}$ et $U_{e}$ les composantes 
connexes de $\overline{\mathbb{C}} \backslash K$. On démontre de la même manière que précédemment que tout point de $K$ tourne à la vitesse $p / q$.

Pour montrer l'assertion relative à l'existence d'une courbe de Jordan invariante, appliquons le lemme 1.1.2 à l'une des composantes connexes de $\overline{\mathbb{C}} \backslash K$ (par exemple $U_{i}$ ). Soit $\mathcal{C}$ une courbe invariante donnée par ce lemme, et $\Omega$ la composante connexe de $\overline{\mathbb{C}} \backslash \mathcal{C}$ telle que $\Omega \subset U_{i}$. Notons $\Omega^{\prime}$ la composante connexe de $\overline{\mathbb{C}} \backslash \mathcal{C}$ contenant $U_{e}$, et $\mathcal{C}^{\prime}=\partial \Omega^{\prime}$. Clairement,

$$
F\left(\Omega^{\prime} \cap V\right) \subset \partial \Omega^{\prime} \quad \text { et } F\left(\mathcal{C}^{\prime}\right)=\mathcal{C}^{\prime} .
$$

Puisque $\mathcal{C}^{\prime} \subset \partial \Omega \cap \partial \Omega^{\prime}$, le complémentaire de $\mathcal{C}^{\prime}$ n'a que deux composantes connexes $\left(\mathcal{C}^{\prime}\right.$ est la réunion d'un nombre fini d'arcs analytiques fermés) et tout point de $\mathcal{C}^{\prime}$ est accessible par chacune de ces deux composantes connexes; on en déduit que $\mathcal{C}^{\prime}$ est une courbe de Jordan.

Ceci termine la démonstration de la proposition 4 .

Appendice : Conjugaison analytique des plongements analytiques de type Morse-Smale. Considérons l'ensemble $\mathcal{S}$ des applications $F$ vérifiant :

(i) Les ensembles de départ (noté $U$ ) et d'arrivée (noté $V$ ) de $F$ sont deux surfaces de Riemann ouvertes et on a $U \subset V$.

(ii) $F$ est un plongement analytique de $U$ dans $V$.

Les éléments de $\mathcal{S}$ seront appelés "plongements analytiques de surfaces de Riemann". Soit $F: U \rightarrow V$ un élément de $\mathcal{S}$. Posons

$$
\begin{gathered}
S_{+}(F)=\bigcap_{k \in \mathbb{N}} F^{-k}(U), \quad S_{-}(F)=\bigcap_{k \in \mathbb{N}} F^{k}(F(U)), \\
S(F)=S_{+}(F) \cap S_{-}(F) .
\end{gathered}
$$

Notons Per $F$ l'ensemble des points périodiques de $F$.

DÉfinition A.1. On dira que $F$ est de type Morse-Smale si les trois conditions suivantes sont remplies :

(i) Per $F$ est fini.

(ii) Tout point de $S_{+}(F)$ (resp. de $S_{-}(F)$ ) converge positivement (resp. négativement) sous l'action de $F$ vers une orbite périodique de $F$.

(iii) Toute orbite périodique indifférente de $F$ a un multiplicateur rationnel.

Remarques. (1) La condition (iii) ci-dessus est en réalité une conséquence de (ii) (on peut donc l'omettre dans cette définition); en effet, au voisinage d'un point fixe indifférent irrationnel par une application holomorphe, il existe toujours des points récurrents non périodiques (voir [PM3]).

(2) On vérifie facilement que, pour toute paire $(K, G)$ appartenant à $\mathcal{K}$ telle que la dynamique de $G$ sur $K$ soit de type Morse-Smale au voisinage 
de $K$ (au sens de la définition 1 de l'introduction), il existe un voisinage $V$ de $K$ tel que $G$ définisse un plongement analytique de $V$ dans $\mathbb{C}$, de type Morse-Smale au sens de la définition A.1 ci-dessus.

On notera $\mathcal{M S}$ l'ensemble des plongements analytiques de type MorseSmale.

DÉfinition A.2. On dira que deux plongements $F: U \rightarrow V$ et $G: U^{\prime} \rightarrow$ $V^{\prime}$ appartenant à $\mathcal{S}$ sont analytiquement conjugués s'il existe un voisinage ouvert $W_{1}$ de $S(F)$ dans $U$, un voisinage ouvert $W_{2}$ de $S(G)$ dans $U^{\prime}$, et un difféomorphisme analytique $\phi$ entre $W_{1}$ et $W_{2}$ tels que :

(i) $\phi\left(W_{1} \cap F^{-1}\left(W_{1}\right)\right)=W_{2} \cap G^{-1}\left(W_{2}\right)$.

(ii) $\phi \circ F(z)=G \circ \phi(z)$ pour tout $z \in W_{1} \cap F^{-1}\left(W_{1}\right)$.

(En particulier $\phi(S(F))=S(G)$, et $\phi$ conjugue $F_{\mid S(F)}$ à $G_{\mid S(G)}$.)

On vérifie que la relation "être analytiquement conjugué" est une relation d'équivalence sur $\mathcal{S}$, et que la propriété d'être de type Morse-Smale est préservée par cette relation d'équivalence.

Nous voudrions maintenant décrire les classes d'équivalence des plongements analytiques de type Morse-Smale pour cette relation.

Cette classification est basée sur le même principe qu'un certain nombre d'autres travaux de classification de dynamiques modulo conjugaison (et s'en inspire) :

- La classification de Mather des difféomorphismes hyperboliques de l'intervalle ([M]).

- La classification d'Écalle-Voronin des germes de difféomorphismes de $(\mathbb{C}, 0)$ tangents à l'identité, considérée du point de vue géométrique ([E], $[\mathrm{V}])$.

- La classification de Palis et Yoccoz des difféomorphismes de type Morse-Smale d'une variété compacte, modulo conjugaison différentiable $([\mathrm{P}, \mathrm{Y}])$.

L'idée principale est due à Mather : on peut tout d'abord exhiber des invariants locaux de conjugaison au voisinage des orbites périodiques (au voisinage d'une orbite périodique attractive ou répulsive, ces invariants locaux sont réduits dans notre cas au multiplicateur de l'orbite). Puis, lorsqu'un bassin attractif et un bassin répulsif s'intersectent, on peut exhiber un invariant de recollement, l' "invariant de Mather", qui décrit la manière dont ces bassins se recollent (dans notre cas, la classification locale au voisinage d'une orbite périodique indifférente va déjà faire intervenir un "invariant de recollement de Mather", puisque nous allons reprendre le point de vue de Voronin dans $[\mathrm{V}])$. 
Il reste alors à montrer que l'ensemble des invariants que l'on a ainsi exhibés est complet, c'est-à-dire que, si deux plongements ont les mêmes invariants, ils sont effectivement analytiquement conjugués; la preuve de ce dernier point est dans notre cas essentiellement triviale, puisque la réunion des bassins attractifs et répulsifs est un ouvert qui contient l'ensemble $S(F)$ au voisinage duquel on veut que la conjugaison soit définie.

Considérons un plongement $F \in \mathcal{S}$ quelconque. Notons $P_{+}$(resp. $P_{-}$) l'ensemble des orbites périodiques attractives (resp. répulsives) par $F$ (attention! chaque élément de $P_{+}$ou de $P_{-}$est une orbite périodique, et non un point d'une orbite périodique).

A toute orbite périodique indifférente par $F$ sont associés un certain nombre de cycles de pétales attractifs ou répulsifs locaux. Notons $P_{0,+}$ (resp. $P_{0,-}$ ) l'ensemble dont les éléments sont les cycles de pétales attractifs (resp. répulsifs) locaux. Posons

$$
\mathcal{P}_{-}=P_{-} \cup P_{0,-}, \quad \mathcal{P}_{+}=P_{+} \cup P_{0,+}, \quad \mathcal{P}=\mathcal{P}_{-} \cup \mathcal{P}_{+} .
$$

A tout élément $p$ de $\mathcal{P}$, on peut naturellement associer un bassin (attractif ou répulsif selon que $p$ appartient à $\mathcal{P}_{+}$ou à $\mathcal{P}_{-}$), que l'on notera $\mathcal{B}(p)$ : par exemple, si $p \in P_{+}, \mathcal{B}(p)$ est l'ensemble des points qui convergent positivement vers l'orbite périodique $p$ (mais qui n'appartiennent pas à $p$ ), et si $p \in P_{0,+}, \mathcal{B}(p)$ est l'ensemble des points dont un itéré appartient au cycle $p$ de petales attractifs locaux. L'ensemble $\mathcal{B}(p)$ est toujours ouvert. Posons

$$
\mathcal{B}_{-}=\coprod_{p \in \mathcal{P}_{-}} \mathcal{B}(p), \quad \mathcal{B}_{+}=\coprod_{p \in \mathcal{P}_{+}} \mathcal{B}(p)
$$

Remarquons que

$S_{-}(F)=\operatorname{Per} F \amalg \mathcal{B}_{-}, \quad S_{+}(F)=\operatorname{Per} F \amalg \mathcal{B}_{+}, \quad S(F)=\operatorname{Per} F \amalg\left(\mathcal{B}_{-} \cap \mathcal{B}_{+}\right)$.

Tout élément $p$ de $\mathcal{P}$ a une période naturelle que l'on note $\operatorname{per}(p)$. Pour tout $p \in P_{+} \cup P_{-}$, notons $\mu(p)$ le multiplicateur de l'orbite périodique $p$ (on a $|\mu(p)|>1$ si $p \in P_{+}$et $|\mu(p)|<1$ si $p \in P_{-}$), et notons $\nu(p)$ la racine $\operatorname{per}(p)$-ième de $\mu(p)$ dont le représentant de l'argument dans $[0 ; 2 \pi[$ est minimal.

Soit $p \in \mathcal{P}_{+}$quelconque. Le bassin attractif $\mathcal{B}(p)$ associé à $p$ admet une partition naturelle en per $(p)$ sous-ensembles qui sont les classes d'équivalence de $\mathcal{B}(p)$ pour la relation d'équivalence suivante $: x \sim y \Leftrightarrow$ il existe $k \in \mathbb{N}$ tel que $F^{k}(x)$ et $F^{k}(y)$ appartiennent à la même composante connexe du cycle de bassins attractifs locaux ou de pétales attractifs locaux. Notons $\mathcal{X}(p)$ l'ensemble (fini, de cardinal per $(p)$ ) dont les éléments sont ces classes d'équivalence; ainsi, 


$$
\mathcal{B}(p)=\coprod_{X \in \mathcal{X}(p)} X
$$

Lorsque $p \in \mathcal{P}_{-}$, on définit $\mathcal{X}(p)$ de la même manière.

Fixons $p \in \mathcal{P}$ quelconque. L'application $F$ agit naturellement sur $\mathcal{X}(p)$, et on notera encore $F$ la permutation de $\mathcal{X}(p)$ définie par cette action.

Notons $l(p)$ l'automorphisme de $\mathbb{C}$ défini par

$$
l(p)(z)= \begin{cases}\nu(p) z & \text { si } p \in P_{-} \cup P_{+}, \\ z+1 / \operatorname{per}(p) & \text { si } p \in P_{0,-} \cup P_{0,+},\end{cases}
$$

et $L(p)$ l'automorphisme de $\mathbb{C} \times \mathcal{X}(p)$ défini par

$$
L(p)(z, X)=(l(p)(z), F(X)) .
$$

Soit $Z(p)$ le groupe de transformations de $\mathbb{C} \times \mathcal{X}(p)$ défini de la manière suivante :

- Si $p \in P_{-} \cup P_{+}, Z(p)$ est constitué des applications $h_{\alpha}:(z, X) \mapsto$ $(\alpha z, X) \alpha \in \mathbb{C}^{*}$ (c'est-à-dire, sur chaque fibre $\mathbb{C}$ au-dessus de $\mathcal{X}(p)$, une homothétie de même rapport).

- Si $p \in P_{0,-} \cup P_{0,+}, Z(p)$ est constitué des applications $T_{\beta}:(z, X) \mapsto$ $(z+\beta, X), \beta \in \mathbb{C}$ (c'est-à-dire, sur chaque fibre $\mathbb{C}$ au-dessus de $\mathcal{X}(p)$, une translation de même vecteur).

Lemme A.1. Pour tout $p \in \mathcal{P}$, il existe une application holomorphe injective $h(p): \mathcal{B}(p) \rightarrow \mathbb{C} \times \mathcal{X}(p)$ telle que, pour tout $z \in \mathcal{B}(p)$, on ait :

(1) Si $X$ désigne l'élément de $\mathcal{X}(p)$ contenant $z$, alors $h(p)(z) \in \mathbb{C} \times X$.

(2) L'application $h(p)$ conjugue $F$ et $L(p)$; plus précisément, si $F(z) \in$ $\mathcal{B}(p)$, alors

$$
h(p) \circ F(z)=L(p) \circ h(p)(z),
$$

et si $F^{-1}(z) \in \mathcal{B}(p)$, alors

$$
h(p) \circ F^{-1}(z)=L(p)^{-1} \circ h(p)(z) .
$$

De plus, l'application $h(p)$ est unique modulo composition à droite par un élément de $Z(p)$.

La démonstration de ce lemme est très simple et très classique lorsque $p \in P_{-} \cup P_{+}$; lorsque $p \in P_{0,-} \cup P_{0,+}$, nous renvoyons à $[\mathrm{V}]$ ou à $[\mathrm{C}, \mathrm{G}]$.

Posons

$$
\mathcal{X}_{-}(F)=\bigcup_{p \in \mathcal{P}_{-}} \mathcal{X}(p), \quad \mathcal{X}_{+}(F)=\bigcup_{p \in \mathcal{P}_{+}} \mathcal{X}(p) .
$$

Remarquons que

$$
\mathcal{B}_{-}=\coprod_{X \in \mathcal{X}_{-}} X, \quad \mathcal{B}_{+}=\coprod_{X \in \mathcal{X}_{+}} X
$$


Posons

$$
\mathcal{V}_{-}(F)=\mathbb{C} \times \mathcal{X}_{-}(F), \quad \mathcal{V}_{+}(F)=\mathbb{C} \times \mathcal{X}_{+}(F)
$$

Soit $Z_{-}(F)$ le groupe d'automorphismes de $\mathcal{V}_{-}(F)$ engendré par l'action de chacun des groupes $Z(p)$ sur $\mathbb{C} \times \mathcal{X}(p)$, pour $p \in \mathcal{P}_{-}$; on définit de même le groupe $Z_{+}(F)$ d'automorphismes de $\mathcal{V}_{+}(F)$.

Soit $h_{-}$le plongement $\mathcal{B}_{-} \rightarrow \mathcal{V}_{-}(F)$ dont la restriction à chacun des $\mathcal{B}(p)$, pour $p \in P_{-}$, coïncide avec $h(p)$. On définit de même le plongement $h_{+}: \mathcal{B}_{+} \rightarrow \mathcal{V}_{+}(F)$. Le plongement $h_{-}$(resp. $\left.h_{+}\right)$est défini de manière unique modulo composition à droite par les éléments de $Z_{-}(F)\left(\right.$ resp. $\left.Z_{+}(F)\right)$.

Notons $\widetilde{\mathcal{I}}(F)$ l'ensemble des triplets $\left(V_{-}, V_{+}, h\right)$ tels que :

(1) $V_{-}$est un ouvert de $\mathcal{V}_{-}(F)$

(2) $V_{+}$est un ouvert de $\mathcal{V}_{+}(F)$.

(3) $h: V_{-} \rightarrow V_{+}$est un difféomorphisme analytique.

Le groupe $Z_{-}(F) \times Z_{+}(F)$ agit naturellement sur $\widetilde{\mathcal{I}}(F)$ de la manière suivante : si $\left(\zeta_{-}, \zeta_{+}\right) \in Z_{-}(F) \times Z_{+}(F)$, on a

$$
\left(\zeta_{-}, \zeta_{+}\right)\left(V_{-}, V_{+}, h\right)=\left(\zeta_{-}\left(V_{-}\right), \zeta_{+}\left(V_{+}\right), \zeta_{+} \circ h \circ \zeta_{-}^{-1}\right) .
$$

Notons $\mathcal{I}(F)$ le quotient de $\tilde{\mathcal{I}}(F)$ par cette action de $Z_{-}(F) \times Z_{+}(F)$. L'élément de $\mathcal{I}(F)$ correspondant à la classe d'équivalence du triplet $\left(h_{-}\left(\mathcal{B}_{+} \cap \mathcal{B}_{-}\right), h_{+}\left(\mathcal{B}_{+} \cap \mathcal{B}_{-}\right), h_{+} \circ h_{-}^{-1}\right)$ pour cette action est défini de manière univoque. Nous le noterons $I(F)$, et nous l'appellerons invariant de recollement associé à $F$.

Considérons à présent deux plongements $F$ et $G$ appartenant à $\mathcal{S}$.

THÉORÈme A.1. Les plongements $F$ et $G$ sont analytiquement conjugués (au sens de la définition A.2) si et seulement si il existe deux applications $\sigma_{-}: \mathcal{X}_{-}(F) \rightarrow \mathcal{X}_{-}(G)$ et $\sigma_{+}: \mathcal{X}_{+}(F) \rightarrow \mathcal{X}_{+}(G)$ satisfaisant aux conditions suivantes :

(1) $\sigma_{+}$(resp. $\left.\sigma_{-}\right)$est bijective, et conjugue l'action de $F$ sur $\mathcal{X}_{+}(F)$ $\left(\right.$ resp. $\left.\mathcal{X}_{-}(F)\right)$ à l'action de $G$ sur $\mathcal{X}_{+}(G)\left(\right.$ resp. $\left.\mathcal{X}_{-}(G)\right)$ (les dynamiques de $F$ et $G$ ont la même combinatoire).

(2) La bijection $\sigma_{+}$met en correspondance des éléments de $\mathcal{X}_{+}(F)$ et $\mathcal{X}_{+}(G)$ de même nature (orbite périodique attractive ou bassin local attractif), et préserve les multiplicateurs des orbites périodiques attractives; la bijection $\sigma_{-}$possède les mêmes propriétés ( $F$ et $G$ ont les mêmes invariants locaux).

Considérons les applications

$$
\begin{gathered}
\widetilde{\sigma}_{+}: \mathcal{V}_{+}(F) \rightarrow \mathcal{V}_{+}(G), \quad(z, X) \mapsto\left(z, \sigma_{+}(X)\right), \\
\tilde{\sigma}_{-}: \mathcal{V}_{-}(F) \rightarrow \mathcal{V}_{-}(G), \quad(z, X) \mapsto\left(z, \sigma_{-}(X)\right), \\
\sigma: \widetilde{\mathcal{I}}(F) \rightarrow \widetilde{\mathcal{I}}(G), \quad\left(V_{-}, V_{+}, h\right) \mapsto\left(\widetilde{\sigma}_{-}\left(V_{-}\right), \widetilde{\sigma}_{+}\left(V_{+}\right), \widetilde{\sigma}_{+} \circ h \circ \widetilde{\sigma}_{-}^{-1}\right) .
\end{gathered}
$$


Grâce à la condition (2) ci-dessus, l'application $\sigma$ passe au quotient et définit une application $\widehat{\sigma}: \mathcal{I}(F) \rightarrow \mathcal{I}(G)$.

(3) On a $\widehat{\sigma}(I(F))=I(G)$.

Démonstration. La partie directe ne pose aucune difficulté. Esquissons la preuve (essentiellement tautologique) de la réciproque.

Notons $U$ et $U^{\prime}$ les domaines de définition respectifs de $F$ et $G$. Considérons des plongements $h_{+}: \mathcal{B}_{+}(F) \rightarrow \mathcal{V}_{+}(F)$ et $h_{-}: \mathcal{B}_{-}(F) \rightarrow \mathcal{V}_{-}(F)$ construits comme plus haut à partir de $F\left(h_{+}\right.$et $h_{-}$sont définis modulo composition à droite par les éléments de $Z_{+}(F)$ et $Z_{-}(F)$ ). De même, considérons des plongements $h_{+}^{\prime}: \mathcal{B}_{+}(G) \rightarrow \mathcal{V}_{+}(G)$ et $h_{-}^{\prime}: \mathcal{B}_{-}(G) \rightarrow \mathcal{V}_{-}(G)$ construits à partir de $G$.

Puisque $\widehat{\sigma}(\mathcal{I}(F))=\mathcal{I}(G)$, on peut supposer, quitte à "corriger" $h_{+}$et $h_{-}$, que

$$
\begin{gathered}
\widetilde{\sigma}_{-} \circ h_{-}(S(F) \backslash \operatorname{Per} F)=h_{-}^{\prime}(S(G) \backslash \operatorname{Per} G), \\
\widetilde{\sigma}_{+} \circ h_{+}(S(F) \backslash \operatorname{Per} F)=h_{+}^{\prime}(S(G) \backslash \operatorname{Per} G), \\
\widetilde{\sigma}_{+} \circ h_{+} \circ h_{-}^{-1}=h_{+}^{\prime} \circ h_{-}^{-1} \quad \operatorname{sur} h_{-}^{\prime}(S(G) \backslash \operatorname{Per} G) .
\end{gathered}
$$

Considérons l'application $\phi: S(F) \backslash \operatorname{Per} F \rightarrow S(G) \backslash \operatorname{Per} G, z \mapsto h_{+}^{\prime-1}$ 。 $\widetilde{\sigma}_{+} \circ h_{+}(z)=h_{-}^{\prime-1} \circ \widetilde{\sigma}_{-} \circ h_{-}^{\prime}(z)$. En utilisant tantôt l'expression $\phi(z)=$ $h_{+}^{\prime-1} \circ \widetilde{\sigma}_{+} \circ h_{+}(z)$ et tantôt $\phi(z)=h_{-}^{\prime-1} \circ \widetilde{\sigma}_{-} \circ h_{-}^{\prime}(z)$, on peut étendre $\phi$ en un difféomorphisme analytique entre un voisinage de $S(F)$ dans $U$ et un voisinage de $S(G)$ dans $U^{\prime}$ qui conjugue $F$ et $G$ (le prolongement de $\phi$ à Per $F$ s'effectue de la même manière que dans $[\mathrm{V}]$ ), ce qui démontre la réciproque.

Le travail effectué ci-dessus va nous permettre également de décrire le centralisateur des éléments de $\mathcal{S}$ (nous adoptons le même point de vue que dans $[\mathrm{P}, \mathrm{Y}])$.

Considérons un plongement $F \in \mathcal{S}$ quelconque. Précisons que nous appelons "centralisateur de $F$ " le groupe des plongements analytiques $\phi$, définis dans un voisinage ouvert $W$ de $S(F)$ (dépendant de $\phi$ ), et vérifiant $\phi(S(F))=S(F)$ et $\phi \circ F=F \circ \phi$ sur $W \cap F^{-1}(W)$.

Reprenons les notations $\mathcal{X}_{-}(F), \mathcal{X}_{+}(F), Z_{-}(F), Z_{+}(F), \tilde{\mathcal{I}}(F)$ introduites plus haut. Considérons le groupe $\Sigma_{-}(F)$ des permutations $\sigma_{-}$de $\mathcal{X}_{-}(F)$ vérifiant les propriétés suivantes :

(1) Les actions de $\sigma_{-}$et de $F \operatorname{sur} \mathcal{X}_{-}(F)$ commutent.

(2) Pour tout $p \in P_{-}(F)$, les multiplicateurs de $p$ et de l'orbite image de $p$ par $\sigma_{-}$coïncident.

De même, on définit un groupe $\Sigma_{+}(F)$ de permutations de $\mathcal{X}_{+}(F)$ vérifiant les mêmes propriétés. 
Les groupes $Z_{-}(F) \times \Sigma_{-}(F)$ et $Z_{+}(F) \times \Sigma_{+}(F)$ agissent naturellement, respectivement sur $\mathbb{C} \times \mathcal{X}_{-}(F)$ et $\mathbb{C} \times \mathcal{X}_{+}(F)$ (voir plus haut), et, de même, le groupe $\left(Z_{-}(F) \times \Sigma_{-}(F)\right) \times\left(Z_{+}(F) \times \Sigma_{+}(F)\right)$ agit naturellement sur l'ensemble $\widetilde{\mathcal{I}}(F)$.

Considérons un représentant $\left(V_{-}, V_{+}, h\right)$ de $I(F)$ dans $\widetilde{\mathcal{I}}(F)$.

DÉfinition. On appellera stabilisateur de l'invariant de $F$ le sousgroupe de $\left(Z_{-}(F) \times \Sigma_{-}(F)\right) \times\left(Z_{+}(F) \times \Sigma_{+}(F)\right)$ constitué des éléments dont l'action sur $\widetilde{\mathcal{I}}(F)$ laisse $\left(V_{-}, V_{+}, h\right)$ fixe (cette définition ne dépend que de $F$, et non du choix de $\left(V_{-}, V_{+}, h\right)$ parmi les représentants de $I(F)$ dans $\widetilde{\mathcal{I}}(F))$.

On a alors la proposition suivante, dont la démonstration ne pose aucune difficulté :

Proposition A.1 (comparer à $[\mathrm{P}, \mathrm{Y}]$, théorème 2). Le centralisateur de $F$ s'identifie canoniquement avec le stabilisateur de l'invariant de $F$.

\section{Bibliographie}

[B] G. D. Birkhoff, Sur quelques courbes fermées remarquables, Bull. Soc. Math. France 60 (1932), 1-26.

[C,G] L. Carleson and T. W. Gamelin, Complex Dynamics, Springer, 1993.

[C,L] M. L. Cartwright and J. C. Littlewood, Some fixed point theorems, Ann. of Math. 54 (1951), 1-37.

[E] J. Ecalle, Théorie des invariants holomorphes, Publ. Math. Orsay 67, 7409 (1974).

[H1] M. R. Herman, Sur la conjugaison différentiable des difféomorphismes du cercle à des rotations, Publ. Math. I.H.E.S. 49 (1979), 5-233.

[H2] - Are there critical points on the boundaries of singular domains?, Comm. Math. Phys. 99 (1985), 593-612.

[L] P. Le Calvez, Propriétés des attracteurs de Birkhoff, Ergodic Theory Dynam. Systems 8 (1987), 241-310.

[M] J. Mather, Commutators of diffeomorphisms, Comm. Math. Helv. 48 (1973), 195-233.

$[\mathrm{P}, \mathrm{Y}] \quad$ J. Palis and J.-C. Yoccoz, Differentiable conjugacies of Morse-Smale diffeomorphisms, Bol. Soc. Brasil. Mat. 20 (1990), 25-48.

[PM1] R. Pérez-Marco, Fixed points and circle maps, Acta Math. 179 (1997), 243294.

[PM2] - Topology of Julia sets and hedgehogs, preprint, Université de Paris-Sud, 94-48, 1994.

[PM3] - Hedgehog's dynamics, preprint.

[PM4] - Classification dynamique des continua pleins invariants par un difféomorphisme holomorphe, manuscrit, 1996.

[Po] C. Pommerenke, Boundary Behaviour of Conformal Maps, Springer, 1992. 
[V] S. M. Voronin, Analytic classification of germs of conformal mappings $(\mathbb{C}, 0) \rightarrow$ $(\mathbb{C}, 0)$ with identity linear part, Funktsional. Anal. i Prilozhen. 15 (1981), no. 1, 1-17 (in Russian).

[Y] J.-C. Yoccoz, Conjugaison des difféomorphismes analytiques du cercle, manuscrit, 1988.

Institut Non Linéaire de Nice

UMR CNRS-UNSA 6618

1361 route des Lucioles

F-06560 Valbonne, France

E-mail: risler@inln.cnrs.fr

Received 14 April 1998;

in revised form 2 March 1999 